\title{
Ultraviolet properties of early-type galaxies ${ }^{\star}$
}

\author{
C. Bonatto ${ }^{1}$, E. Bica ${ }^{1}$, M.G. Pastoriza ${ }^{1}$ and D. Alloin ${ }^{2,3}$ \\ 1 Universidade Federal do Rio Grande do Sul, IF, CP 15051, Porto Alegre 91501-970, RS, Brazil \\ 2 Observatoire de Paris/Meudon, URA 173 CNRS, F-92195 Meudon Principal Cedex, France \\ 3 Canada-France-Hawaii Telescope, P.O. box 1597, Kamuela, Hawaii - 96743, U.S.A.
}

Received October 9; accepted December 21, 1995

\begin{abstract}
We analyse the UV properties of early-type galaxies from their UV spectra in the IUE library, including both normal and active nuclei. We co-added the spectra, and hence the objects into groups of similar spectral properties in the UV, also taking into account their properties in the visible/near-infrared ranges. Although, owing to the presence of a residual fixed pattern noise, IUE data cannot be improved by co-addition as expected for spectra containing only random noise, this procedure still provided spectra of higher signal/noise ratio than in previous studies, often based on individual spectra and therefrom derived colour indices. Thanks to the co-adding procedure, information on spectral features can now be assessed. The red stellar population groups exhibit a far-UV flux at different levels. The higher ones denote the presence of the UV turnup, which possibly exhibits absorption features, at least as detected through the IUE aperture. As to the blue stellar population groups and/or AGNs, we have carried out simple syntheses with UV star cluster templates and galaxy spectra in order to infer the properties such as burst ages and contamination by an active nucleus. It has been possible to derive information on the extinction law affecting some internally reddened galaxies; there are cases where the presence of the $\lambda 2200 \AA$ absorption feature suggests a reddening law similar to the Galactic one, and others without the $\lambda 2200 \AA$ feature, suggesting that it is rather an SMC type law which applies, then.
\end{abstract}

Key words: (ISM:) dust, extinction — galaxies: active — galaxies: elliptical and lenticular, cD — galaxies: general — galaxies: stellar content — ultraviolet: galaxies

\section{Introduction}

The International Ultraviolet Explorer (IUE) Satellite has been collecting fundamental data on a wide variety of astronomical objects since 1978. Recently we have used the IUE library to study the spectral properties of a large sample of integrated star cluster spectra with an unprecedented signal to noise $(S / N)$ ratio by co-adding them into groups. The grouping procedure allowed us to analyse in detail the far-UV properties of star clusters and to provide reliable information on absorption features. The results of this analysis were presented in Bonatto et al. (1995, hereafter Paper I).

Regarding galaxies, several studies of IUE spectra by object class have already been carried out, e.g. the spectral atlases by Kinney et al. (1993) for star-forming galaxies, and by Rosa et al. (1984) for H II complexes.

In this paper we gather the spectra of the early-type galaxies stored in the IUE database and analyse the ensembles of normal and active early-type galaxies, in view

Send offprint requests to: D. Alloin

*Based upon data collected with the International Ultraviolet Explorer (IUE) Satellite, supported by NASA, SERC and ESA of better understanding their far-UV spectral properties. We also use, for the interpretation, single-aged UV templates of stellar populations, i.e. the star cluster spectral groups from Paper I, which help constrain the conclusions.

Some normal early-type galaxies (in this paper, ellipticals and S 0's) are known to emit large amounts of energy in the far-UV, producing the so-called UV turnup (e.g. Bertola et al. 1980; Oke et al. 1981). However, the characteristics and origin of this emission are not yet fully understood. So far, studies of these objects have been performed from individual IUE spectra which usually have a rather low $(S / N)$ ratio, or have been based on colour indices (Burstein et al. 1988; Longo et al. 1991). Bica \& Alloin (1988) have used interpolated continuum distribution.

The analyses of IUE spectra of Active Galactic Nuclei (AGNs) in early-type galaxies have been mostly focused on variability, e.g. NGC 3516 (Wanders et al. 1993) and Mk 279 (Stirpe et al. 1994). Our goal here is to study time average properties by co-adding spectra of a given AGN, then classifying and co-adding the average spectra into groups of comparable spectral characteristics. So we work with the average activity state in which each AGN was observed. 
This paper is organised as follows: in Sect. 2 we present the IUE data on early-type galaxies. In Sect. 3 we group the objects according to spectral similarities from the UV to the visible/near-IR ranges. Equivalent width and continuum measurements are presented in Sect. 4 . In Sect. 5 we discuss spectral groups as well as some interesting individual objects. Concluding remarks are provided in Sect. 6 .

\section{The data}

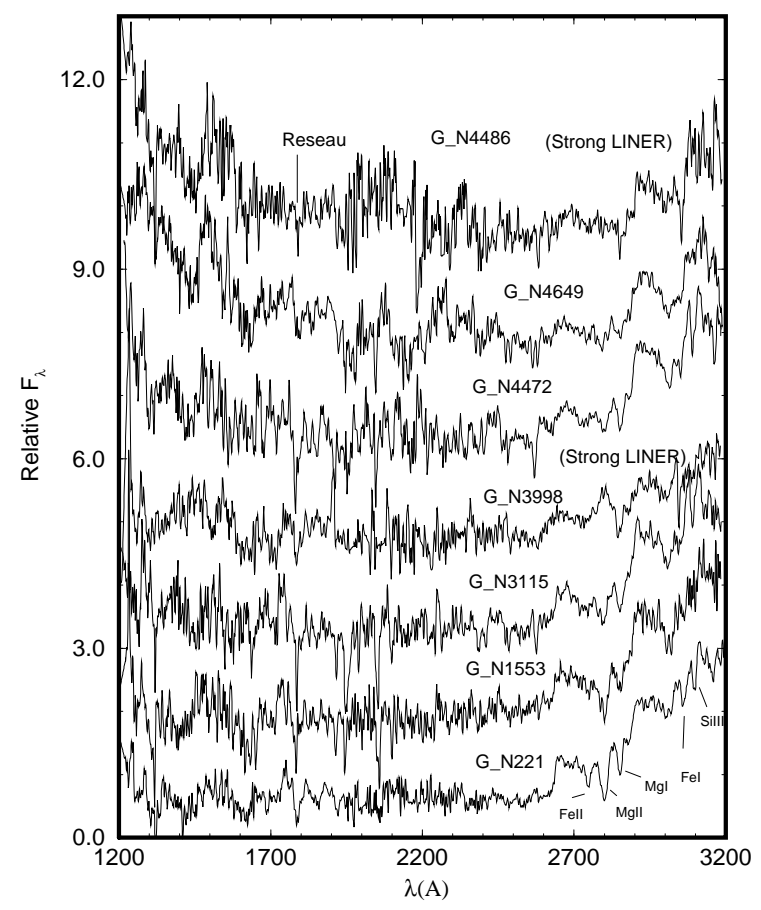

Fig. 1. Groups for red stellar populations in early-type galaxies with different contributions from the UV turnup. Flux in $F_{\lambda}$ units, normalised at $\lambda 2646 \AA$. Constants have been added to the spectra for clarity purposes, except for the bottom one

The spectra have been extracted from the IUE database as stored at the Instituto Astronômico e Geofísico of the Universidade de São Paulo (IAG-USP). We have selected the available SWP, LWP and LWR spectra of early-type galaxies obtained in the large aperture $\left(10^{\prime \prime} \times 20^{\prime \prime}\right)$ mode. In Table 1 we present the objects used in this study along with some relevant data. Only objects with a morphological type $T<0$ have been used (de Vaucouleurs et al. 1991, hereafter RC3). We also included NGC 5253 which was classified as a peculiar earlytype galaxy $(T=10)$ in the RC3 (see also Sérsic et al. 1972). We use as well in the discussion IUE spectra with high $(S / N)$ ratio for some control galaxies, which are NGC 5236 (nuclear starburst in a spiral galaxy; Pastoriza 1975), NGC 1068 (Seyfert 2 in a spiral galaxy), NGC 4151 (Seyfert 1 in late-type spiral) and NGC 7213 (Seyfert 1/LINER in an early-type spiral). We have gath-

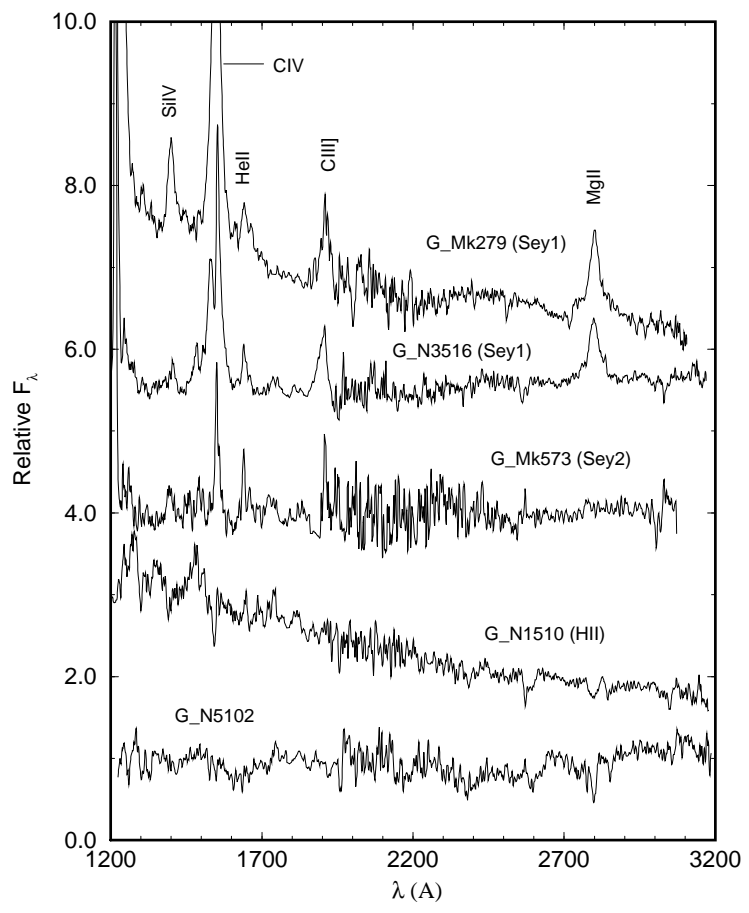

Fig. 2. Groups for AGN together with groups for blue stellar populations in early-type galaxies. Notice how the two groups of Seyfert 1s have similar emission lines while diverging in the continuum slope. Flux in $F_{\lambda}$ units, normalised at $\lambda 2646 \AA$. Constants have been added to the spectra for clarity purposes, except for the bottom one

ered in Table 1 the following information on the early-type sample galaxies, by columns: (1) different identifications; (2) PGC number; (3) morphological type (T); (4) foreground absorption $A_{B} ;(5)$ total blue magnitude $B_{T} ;(6)$ $M_{B}$ assuming $H_{0}=75 \mathrm{~km} \mathrm{~s}^{-1} \mathrm{Mpc}^{-1}$; (7) heliocentric radial velocity, and (8) complementary remarks. Absolute magnitudes $M_{B}$ were calculated using Galactic Standard of Rest $\left(V_{\mathrm{G}}\right)$ velocities (RC3), while optical and H I heliocentric velocities $\left(V_{R}\right)$ were considered for redshift corrections in the IUE spectra. The main sources (and references therein) were: RC3 (identifications, PGC number, $T, V_{R}$, $A_{B}, B, V_{\mathrm{G}}$ for $M_{B}$ calculations); Sandage \& Tammann (1981) for $V_{G}$ in some groups of galaxies; Mazzarella \& Balzano (1986) for data on Markarian galaxies; Véron \& Véron (1991) and Hewitt \& Burbidge (1993) for data on AGNs. Additional relevant references are given in Sect. 3. The $A_{B}$ values were used to correct all spectra for the foreground (Galactic) reddening using Seaton's (1979) law and $A_{B} / E(B-V)=4.0$. Thus, in the subsequent analyses all spectra are corrected for the foreground reddening and redshift. 
Table 1. Early-type galaxies

\begin{tabular}{|c|c|c|c|c|c|c|c|}
\hline Object & $\begin{array}{c}\text { PGC } \\
\#\end{array}$ & $T$ & $\begin{array}{c}A_{B} \\
\text { mag }\end{array}$ & $\begin{array}{c}B_{T} \\
\mathrm{mag}\end{array}$ & $\begin{array}{l}M_{B} \\
\mathrm{mag}\end{array}$ & $\begin{array}{c}V_{R} \\
\mathrm{~km} \mathrm{~s}^{-1}\end{array}$ & Remarks \\
\hline ESO 252-G18 & 17116 & -2.0 & 0.10 & 15.94 & -19.8 & 10172 & Seyfert 1, Radio Galaxy \\
\hline ESO 434-G40, MCG-05-23-0016 & 28144 & $*$ & 0.49 & 13.54 & -19.5 & 2482 & Seyfert 2 \\
\hline IC 450, Mk6, UGC 3547, ARP 121 & 19756 & -0.5 & 0.36 & 14.48 & -20.2 & 5440 & Seyfert 2 \\
\hline IC 1065, UGC 9553, 3C 305 & 52924 & -2.0 & 0.03 & 14.43 & -21.7 & 12423 & Radio Galaxy \\
\hline IC 3370, ESO 322-G14, IRAS 100469-3903 & 40887 & -5.0 & 0.35 & 11.59 & -21.7 & 2930 & in cluster \\
\hline IC 4296, ESO 383-G39, MCG-06-30-0016 & 48040 & -5.0 & 0.13 & 11.42 & -22.2 & 3727 & Radio Galaxy, in cluster \\
\hline IC 4329A, ESO 445-G50, MCG-05-33-0021 & 49051 & -0.7 & 0.15 & 13.67 & -20.5 & 4762 & Seyfert 1 , strong dust lane \\
\hline IC 5063, ESO 187-G23, IRAS 20481-5715 & 65600 & -0.8 & 0.25 & 12.58 & -21.0 & 3400 & Seyfert 2 \\
\hline MCG+08-31-0041, ARP 102B, UGC 10814 & 60067 & -5.0 & 0.07 & 14.94 & -20.0 & 7172 & Seyfert 1 \\
\hline M 32, NGC 221, UGC 452, Ark 12, ARP 168 & 2555 & -6.0 & 0.33 & 8.72 & -15.7 & 186 & in Local group \\
\hline M 49, NGC 4472, UGC 7629, ARP 134 & 41220 & -5.0 & 0.00 & 9.33 & -21.4 & 983 & H II galaxy, in Virgo cluster \\
\hline M 59, NGC 4621, UGC 7858, VCC 1903 & 42628 & -5.0 & 0.07 & 10.53 & -20.2 & 444 & in Virgo cluster \\
\hline M 60, NGC 4649, UGC 7898, in ARP 116 & 42831 & -5.0 & 0.04 & 9.70 & -21.0 & 1114 & in Virgo cluster \\
\hline M 84, NGC 4374, UGC 7494, VCC 763, 3C 272.1 & 40455 & -5.0 & 0.13 & 10.01 & -20.8 & 1098 & in Virgo cluster \\
\hline M 85, NGC 4382, UGC 7508, VCC 798 & 40515 & -1.0 & 0.03 & 9.99 & -20.7 & 754 & in Virgo cluster \\
\hline M 86, NGC 4406, UGC 7532, VCC 881 & 40653 & -5.0 & 0.12 & 9.74 & -21.1 & -248 & in Virgo cluster \\
\hline $\begin{array}{l}\text { M } 87, \text { NGC } 4486, \text { UGC } 7654, \text { ARP } 152, \\
\text { 3C } 274.0, \text { Vir A }\end{array}$ & 41361 & -4.0 & 0.10 & 9.49 & -21.3 & 1282 & $\begin{array}{l}\text { Strong LINER, Radio Galaxy, } \\
\text { in Virgo cluster }\end{array}$ \\
\hline M 89, NGC 4552, UGC 7760 , VCC 1632 & 41968 & -5.0 & 0.15 & 10.57 & -20.3 & 397 & in Virgo cluster \\
\hline M 105, NGC 3379, UGC 5902 & 32256 & -5.0 & 0.05 & 10.18 & -20.3 & 889 & \\
\hline Mk 9, Haro 1, CGCG286- 36 & 21400 & -2.0 & 0.14 & 14.97 & -21.1 & 11587 & Seyfert 1 \\
\hline Mk 49=Mk 1318, Haro 8, UGC 7354 & 39628 & -5.0 & 0.02 & 14.44 & -17.3 & 1684 & $\mathrm{H} \mathrm{II}$ \\
\hline Mk 198, MCG+08-22-0073 & 38613 & -2.0 & 0.00 & 14.86 & -20.0 & 7084 & Seyfert 2 \\
\hline Mk 279, UGC 8823 & 49321 & -2.0 & 0.00 & 14.43 & -20.9 & 8778 & Seyfert 1 , in pair \\
\hline Mk 290, QSO1534+580 & 55551 & -5.0 & 0.01 & 15.07 & -20.3 & 8690 & Seyfert 1 \\
\hline Mk 352, IRAS $0057+316$ & 3575 & -2.0 & 0.24 & 14.49 & -19.6 & 4437 & Seyfert 1 \\
\hline Mk 573, UM 363, UGC 1214 & 6367 & -1.0 & 0.03 & 13.57 & -20.7 & 5175 & Seyfert 2 , in pair \\
\hline Mk 705, UGC 5025, Ark 202, VIIIZw 47 & 26753 & -2.0 & 0.08 & 14.50 & -20.9 & 8545 & Seyfert 1 \\
\hline Mk 960, Haro 15, MCG-02-03-0019 & 2845 & -2.0 & 0.09 & 13.90 & -20.5 & 5359 & H II galaxy, in system \\
\hline Mk 1018, UM 393, UGC 1597 & 8029 & -2.0 & 0.01 & 14.10 & -21.9 & 12077 & Seyfert 1 \\
\hline $\begin{array}{l}\text { Mk } 1506, \text { UGC 3087, IIZw 14, } \\
\quad \text { IRAS } 0435+0514, \text { MCG +01-12-0009 }\end{array}$ & 15504 & -2.0 & 0.61 & 14.20 & -21.9 & 9528 & Seyfert 1 \\
\hline NGC 185, UGC 396, IRAS 00362+4803 & 2329 & -5.0 & 0.83 & 9.28 & -15.7 & -251 & in Local group \\
\hline NGC 205, UGC 426, IRAS 00376+4124 & 2429 & -5.0 & 0.15 & 8.79 & -15.5 & -344 & in Local group \\
\hline NGC 244, MCG-03-03-0003, VV 728 & 2675 & -1.7 & 0.02 & 13.00 & -17.4 & 944 & \\
\hline NGC 404, UGC 718, IRAS 01066+3527 & 4126 & -3.0 & 0.24 & 10.92 & -14.3 & -19 & in Local group? \\
\hline NGC 584, MCG-01-04-0060 & 5663 & -5.0 & 0.14 & 11.21 & -20.9 & 1864 & \\
\hline NGC 936, UGC 1929 & 9359 & -1.0 & 0.05 & 10.98 & -20.4 & 1400 & \\
\hline NGC 1023, UGC 2154, ARP 135 & 10123 & -3.0 & 0.27 & 10.08 & -19.7 & 601 & \\
\hline NGC 1052, MCG-01-07-0034, IRAS 02386-0828 & 10175 & -5.0 & 0.06 & 11.35 & -20.2 & 1474 & Strong LINER \\
\hline NGC 1106, UGC 2322, IRAS $02474+4127$ & 10792 & -1.0 & 0.32 & 12.92 & -21.1 & 4205 & Seyfert 2 \\
\hline NGC 1316, ESO 357-G22, ARP 154, For A & 12651 & -2.0 & 0.00 & 9.40 & -22.1 & 1172 & Radio Galaxy, in Fornax cluster \\
\hline NGC 1326, ESO 357-G26, IRAS 03220-3638 & 12709 & -1.0 & 0.00 & 11.39 & -20.1 & 415 & in Fornax cluster \\
\hline NGC 1399, ESO 358-G45 & 13418 & -5.0 & 0.00 & 10.44 & -21.0 & 1483 & in Fornax cluster \\
\hline NGC 1404, ESO 358-G46 & 13433 & -5.0 & 0.00 & 10.98 & -20.5 & 1483 & in Fornax cluster \\
\hline NGC 1407, ESO 548-G67 & 13505 & -5.0 & 0.17 & 10.71 & -21.3 & 1762 & \\
\hline NGC 1510 , ESO 250-G3, IRAS 04019-4332 & 14375 & -2.3 & 0.00 & 13.45 & -17.1 & 989 & H II galaxy, in pair \\
\hline NGC 1533, ESO 157-G3, IRAS 04088-5614 & 14582 & -3.0 & 0.00 & 11.74 & -18.0 & 668 & in group \\
\hline NGC 1553, ESO 157-G17, IRAS 04150-5554 & 14765 & -2.0 & 0.00 & 10.26 & -20.8 & 1239 & in group \\
\hline NGC 1705, ESO 158-G13, IRAS 04531-5326 & 16282 & -3.0 & 0.19 & 12.58 & -15.2 & 252 & H II galaxy \\
\hline NGC 1947, ESO 85-G87, IRAS 05264-6347 & 17296 & -3.0 & 0.16 & 11.50 & -19.6 & 1157 & Strong dust lane \\
\hline NGC 2110, MCG-01-15-0004 & 18030 & -3.0 & 1.56 & 14.00 & -20.0 & 2284 & Seyfert 2 \\
\hline NGC 2217, ESO 489-G42, IRAS 06196-2712 & 18883 & -1.0 & 0.15 & 11.35 & -20.5 & 1609 & \\
\hline NGC 2768, UGC 4821 & 25915 & -5.0 & 0.17 & 10.70 & -20.7 & 1335 & \\
\hline NGC 2784, ESO 497-G23, AM 0910-235 & 25950 & -2.0 & 0.79 & 10.46 & -20.1 & 691 & \\
\hline NGC 2865, ESO 498-G1 & 26601 & -5.0 & 0.29 & 12.18 & -20.8 & 2611 & \\
\hline NGC 3115, MCG-01-26-0018 & 29265 & -3.0 & 0.10 & 9.74 & -20.1 & 670 & \\
\hline NGC 3125, ESO 435-G41, IRAS 10042-2941 & 29366 & -5.0 & 0.27 & 13.11 & -17.9 & 1080 & H II galaxy, in cluster \\
\hline NGC 3156, UGC 5503 & 29730 & -2.0 & 0.04 & 12.91 & -18.0 & 1118 & \\
\hline NGC 3311, ESO 501-G38 & 31478 & -4.0 & 0.18 & 12.22 & -21.5 & 3756 & \\
\hline
\end{tabular}

Table Notes. $\left(^{*}\right)$ Lauberts (1982) classified this galaxy as S 0.

\section{Spectral groups}

Galaxies exhibit a wide range of spectral properties, but those presenting similarities can be grouped, a procedure which allows one to study their average properties in more detail, from data with an improved $(S / N)$ ratio. In order to obtain such data and to analyse the UV spectral lines it is necessary first to co-add the available IUE spectra of each individual object, and subsequently to coadd into a spectral group the spectra of objects which show close similarities. In the present grouping procedure the ultimate criterion is the similarity of the UV spectra at the available $(S / N)$ ratio. However, we also consider the spectral properties in the visible and near-IR ranges found in the literature, together with the classifications as AGN, HII galaxy, starburst or usual normal stellar 
Table 1. continued

\begin{tabular}{|c|c|c|c|c|c|c|c|}
\hline Object & $\begin{array}{l}\text { PGC } \\
\#\end{array}$ & $\mathrm{~T}$ & $\begin{array}{c}\mathrm{A}_{B} \\
\mathrm{mag}\end{array}$ & $\begin{array}{c}\mathrm{B}_{T} \\
\mathrm{mag}\end{array}$ & $\begin{array}{l}\mathrm{M}_{B} \\
\mathrm{mag}\end{array}$ & $\begin{array}{c}\mathrm{V}_{R} \\
\mathrm{~km} \mathrm{~s}^{-1}\end{array}$ & Remarks \\
\hline NGC 3516, UGC 6153, IRAS 11033+7250 & 33623 & -2.0 & 0.10 & 12.14 & -20.7 & 2614 & Seyfert 1 \\
\hline NGC 3894, UGC 6779 & 36889 & -5.0 & 0.00 & 12.60 & -20.5 & 3193 & \\
\hline NGC 3998, UGC 6946, MCG+09-20-0046 & 37642 & -2.0 & 0.00 & 11.49 & -19.3 & 1066 & Strong LINER \\
\hline NGC 4111, UGC 7103 & 38440 & -1.0 & 0.00 & 11.60 & -18.6 & 815 & \\
\hline NGC 4125, UGC 7118 , IRAS $12055+6527$ & 38524 & -5.0 & 0.04 & 10.67 & -20.6 & 1356 & \\
\hline NGC 4278 , UGC 7386 , IRAS $12175+2933$ & 39764 & -5.0 & 0.11 & 10.97 & -18.8 & 628 & Strong LINER, Radio Galaxy \\
\hline NGC 4350, UGC 7473, VCC 685, Ark 362 & 40295 & -2.0 & 0.03 & 11.93 & -18.8 & 1158 & in Virgo cluster \\
\hline NGC 4385, Mk 52, UGC 7515, UM 499 & 40564 & -1.0 & 0.03 & 12.90 & -19.5 & 2271 & H II galaxy \\
\hline NGC 4494, UGC 7662 & 41441 & -5.0 & 0.06 & 10.55 & -20.7 & 1324 & \\
\hline NGC 4564, UGC 7773, VCC 1664 & 42051 & -5.0 & 0.09 & 11.96 & -18.8 & 1119 & in Virgo cluster \\
\hline NGC 4696, ESO 322-G91 & 43296 & -4.0 & 0.50 & 10.85 & -22.6 & 2958 & in cluster \\
\hline NGC 4697, MCG-01-33-0010 & 43276 & -5.0 & 0.04 & 10.07 & -21.0 & 1236 & \\
\hline NGC 4742, MCG-02-33-0032, IRAS 12490-1009 & 43594 & -5.0 & 0.10 & 11.94 & -19.3 & 1286 & \\
\hline NGC 4762, UGC 8016, VCC 2095 & 43733 & -2.0 & 0.00 & 11.01 & -19.9 & 971 & in Virgo cluster \\
\hline NGC 4853, UGC 8092, 2Zw 67 & 44481 & -3.0 & 0.04 & 14.23 & -20.8 & 7494 & in Coma cluster \\
\hline NGC 4874, UGC 8103 & 44628 & -4.0 & 0.05 & 12.61 & -22.3 & 7055 & in Coma cluster \\
\hline NGC 4889, UGC 8110 & 44715 & -4.0 & 0.05 & 12.34 & -22.4 & 6410 & in Coma cluster \\
\hline NGC 5044, MCG-03-34-0034 & 46115 & -5.0 & 0.12 & 11.67 & -21.2 & 2692 & \\
\hline NGC 5102, ESO 382-G50, IRAS 13191-3622 & 46674 & -3.0 & 0.22 & 9.99 & -17.9 & 715 & in Centaurus group \\
\hline NGC 5128, ESO 270-IG9, ARP 153, CenA & 46957 & -2.0 & 0.24 & 7.30 & -20.6 & 595 & $\begin{array}{l}\text { Strong dust lane, Radio Galaxy } \\
\text { in Centaurus group }\end{array}$ \\
\hline $\begin{array}{l}\text { NGC } 5253, \text { ESO } 445-G 4, \text { Haro } 10 \\
\text { IRAS } 13370-3123\end{array}$ & 48334 & 10.0 & 0.20 & 10.47 & -17.4 & 547 & in Centaurus group \\
\hline NGC 5266, ESO 220-G33, IRAS 13399-4755 & 48593 & -3.0 & 0.53 & 11.50 & -22.1 & 3044 & in group \\
\hline NGC 5273, UGC 8675, MCG+06-30-0072 & 48521 & -2.0 & 0.00 & 12.38 & -18.3 & 1054 & Seyfert 1 \\
\hline NGC 5283, Mk 270, UGC 8672 & 48425 & -2.0 & 0.04 & 14.12 & -19.3 & 3578 & Seyfert 2 \\
\hline NGC 5670, ESO 272-G19 & 52161 & -2.0 & 0.66 & 12.25 & -21.3 & 2866 & \\
\hline NGC 5813, UGC 9655 & 53643 & -5.0 & 0.16 & 11.42 & -20.8 & 1924 & \\
\hline NGC 5846, UGC 9706 & 53932 & -5.0 & 0.15 & 10.91 & -21.0 & 1710 & \\
\hline NGC 6166, UGC 10409, VV 364 & 58265 & -4.0 & 0.00 & 12.83 & -22.6 & 8952 & $\mathrm{cD}$ \\
\hline NGC 6868, ESO 233-G39 & 64192 & -5.0 & 0.15 & 11.49 & -21.5 & 2836 & in pair \\
\hline NGC 7196, ESO 237-G36, IRAS 22026-5021 & 68020 & -5.0 & 0.00 & 12.35 & -20.6 & 2896 & in group \\
\hline NGC 7385, UGC 12207 & 69824 & -5.0 & 0.16 & 13.12 & -22.1 & 7699 & Radio Galaxy \\
\hline NGC 7465, Mk 313, UGC 12317, Ark 573 & 70295 & -1.5 & 0.21 & 13.07 & -19.3 & 1999 & Seyfert 2 \\
\hline
\end{tabular}

population types. In order to do this we have used the information from references quoted in Sect. 2 and the following ones, which were useful for classification according to stellar population and/or emitting gas characteristics: Heckman et al. (1980); Caldwell (1984); Dahari (1985); Filippenko \& Sargent (1985); Bica \& Alloin (1987a,b); Davies et al. (1987); Bettoni \& Buson (1987); Gregg (1989); Bonatto et al. (1989); Terlevich et al. (1991); Bica et al. (1991); Buson et al. (1993); Osterbrock \& Martel (1993); Goudfrooij et al. (1994); Gelderman \& Whittle (1994); Storchi-Bergmann et al. (1995).

Three steps were taken to build up the final spectral groups: i) we averaged individual IUE spectra of a given object working separately in the short (SWP) and long (LWP and LWR) wavelength ranges. A few spectra were eliminated at this stage because they presented problems such as instrumental defects, discordant continuum distribution and/or spectral features; ii) we co-added spectra of different objects with comparable spectral properties the ultimate criterion being the spectral similarity in the UV; and iii) the short and long wavelength domains were finally connected. The weight of each spectrum in the average was attributed according to the square of the $(S / N)$ ratio.

In Tables ?? and ?? we present the resulting groups with the galaxies they are made of, along with the num- ber of SWP and LWP/LWR spectra effectively used for each galaxy. The spectral group is named after a member which has spectra both in the SWP and LWP/LWR domains with a good $(S / N)$ ratio. The projected IUE aperture in kpc is shown in Tables ?? and ?? for individual galaxies as well as its mean value for the group. We also recall in Tables ?? and ?? the spectral groups for the nuclear stellar population of early-type galaxies from the visible and near-IR ranges for which Bica (1988) carried out population syntheses: E 1 to E 4 are nuclear red stellar populations in a decreasing metallicity sequence; E 5 is as strong-lined as E 1 and E 2, but appears to have a somewhat stronger intermediate age component; E 7 exhibits an intermediate age burst of star formation and $\mathrm{E} 8$ had a burst $\approx 500$ Myr ago. NGC 1533 and NGC 1316 were grouped with spirals in Bica (1988) following the morphological classifications of Sandage \& Tammann (1981) as SB 0/SB a and S a peculiar respectively, resulting in the labels of nuclear stellar population spectral groups S 1 (similar to E 1) and S 2 (similar to E 2), respectively.

Groups of red stellar population in early-type galaxies are displayed in Fig. ??. Groups with active nuclei together with groups of blue stellar population in early-type galaxies are shown in Fig. ??. The IUE $\lambda 1790 \AA$ réseau mark indicated in Fig. ?? has been clipped out in the subsequent figures. 
Table 2. Red stellar population spectral groups

\begin{tabular}{|c|c|c|c|c|}
\hline Galaxy & SWP & $\mathrm{LWP} / \mathrm{R}$ & $\begin{array}{c}\text { slit } \\
(\mathrm{kpc} \times \mathrm{kpc})\end{array}$ & $\begin{array}{l}\text { Spectral } \\
\text { Type }\end{array}$ \\
\hline \multicolumn{5}{|c|}{ With intermediate age burst } \\
\hline & \multicolumn{2}{|c|}{ G_N221 } & $\langle 0.7 \times 1.5\rangle$ & \\
\hline$\overline{\text { NGC } 221}$ & 5 & 5 & $0.03 \times 0.06$ & \\
\hline NGC 2865 & 1 & 1 & $1.7 \times 3.4$ & G-E 7 \\
\hline NGC 4111 & 2 & 2 & $0.5 \times 1.1$ & \\
\hline NGC 4382 & 1 & 1 & $0.7 \times 1.4$ & $\mathrm{G}-\mathrm{E} 7$ \\
\hline NGC 4742 & 2 & - & $0.8 \times 1.7$ & $\mathrm{G}-\mathrm{E} 7$ \\
\hline \multicolumn{5}{|c|}{ UV weak } \\
\hline & \multicolumn{2}{|c|}{ G_N1553 } & $\langle 1.2 \times 2.4\rangle$ & \\
\hline$\overline{\mathrm{NGC} 244}$ & - & 1 & $0.6 \times 1.2$ & \\
\hline NGC 584 & 1 & - & $1.2 \times 2.4$ & G-E 5 \\
\hline NGC 1316 & 1 & 1 & $0.8 \times 1.5$ & $\mathrm{G}-\mathrm{S} 2$ \\
\hline NGC 1553 & 2 & 1 & $0.8 \times 1.6$ & G-E 5 \\
\hline NGC 3311 & 1 & - & $2.4 \times 4.9$ & \\
\hline NGC 4494 & 1 & - & $0.9 \times 1.7$ & \\
\hline NGC 4696 & 2 & - & $1.9 \times 3.8$ & \\
\hline NGC 4697 & 2 & 1 & $0.8 \times 1.6$ & G-E 5 \\
\hline NGC 4762 & 1 & 3 & $0.7 \times 1.4$ & \\
\hline NGC 5044 & 1 & - & $1.7 \times 3.5$ & \\
\hline NGC 5846 & 1 & - & $1.1 \times 2.2$ & \\
\hline \multirow[t]{3}{*}{ NGC 6868} & 1 & - & $1.8 \times 3.7$ & G-E 5 \\
\hline & \multicolumn{3}{|c|}{ UV weak } & \\
\hline & \multicolumn{2}{|c|}{ G_N3115 } & $\langle 0.9 \times 1.8\rangle$ & \\
\hline$\overline{\mathrm{IC}} 3370$ & 1 & - & $1.9 \times 3.8$ & \\
\hline NGC 1023 & - & 1 & $0.4 \times 0.9$ & \\
\hline NGC 1533 & - & 1 & $0.4 \times 0.9$ & $\mathrm{G}-\mathrm{S} 1$ \\
\hline NGC 2768 & 1 & 1 & $0.9 \times 1.7$ & \\
\hline NGC 3115 & 2 & 3 & $0.4 \times 0.9$ & $\mathrm{G}-\mathrm{E} 2$ \\
\hline NGC 3379 & 2 & 2 & $0.6 \times 1.2$ & $\mathrm{G}-\mathrm{E} 2$ \\
\hline NGC 4125 & 1 & 1 & $0.9 \times 1.8$ & \\
\hline NGC 4350 & 2 & 3 & $0.7 \times 1.4$ & \\
\hline NGC 4564 & 1 & - & $0.7 \times 1.4$ & \\
\hline NGC 5670 & 1 & 1 & $1.9 \times 3.7$ & \\
\hline NGC 5813 & - & 1 & $1.3 \times 2.5$ & \\
\hline
\end{tabular}

Table Notes. Cols. 2 and 3 give the number of IUE spectra effectively used; Col. 4 gives the projected IUE aperture $\left(10^{\prime \prime} \times 20^{\prime \prime}\right)$ in $\mathrm{kpc} \times \mathrm{kpc}$.

\section{Measurements}

In Paper I we defined a series of windows and regions in the UV which are useful for measuring equivalent widths (W) and continuum points. In Table 4 we present the Ws for the UV windows measured in the blue and red stellar population groups, as well as in some sample individual galaxies. The number and wavelength limits of the UV windows (as defined in PaperI), together with the main absorbers in each window, are given in the first three columns of Table 4 , respectively.

Continuum points, normalised at $\lambda 2646 \AA$, are provided in Table 5 , where we have also included the Seyfert groups and their respective control galaxies. The quoted
Table 2. continued

\begin{tabular}{|c|c|c|c|c|}
\hline Galaxy & SWP & $\mathrm{LWP} / \mathrm{R}$ & $\begin{array}{c}\text { slit } \\
(\mathrm{kpc} \times \mathrm{kpc})\end{array}$ & $\begin{array}{c}\text { Spectral } \\
\text { Type }\end{array}$ \\
\hline \multicolumn{5}{|c|}{ Strong LINER } \\
\hline & \multicolumn{2}{|c|}{ G_N3998 } & \multicolumn{2}{|l|}{$\langle 3.1 \times 6.2\rangle$} \\
\hline $\begin{array}{l}\text { NGC } 3998 \\
\end{array}$ & 6 & 6 & $0.7 \times 1.4$ & \\
\hline NGC 4278 & 1 & 1 & $0.4 \times 0.8$ & \\
\hline IC 1065 & 1 & 1 & $8.1 \times 16.1$ & \\
\hline \multicolumn{5}{|c|}{ Moderate UV turnup } \\
\hline & \multicolumn{2}{|c|}{ G_N4472 } & $\langle 1.0 \times 2.0\rangle$ & \\
\hline$\overline{\text { NGC } 936}$ & - & 1 & $0.9 \times 1.8$ & \multirow{4}{*}{ G-E 5} \\
\hline NGC 1404 & 4 & 3 & $1.0 \times 1.9$ & \\
\hline NGC 1407 & 4 & - & $1.1 \times 2.3$ & \\
\hline NGC 2217 & - & 3 & $1.0 \times 2.1$ & \\
\hline NGC 4374 & 2 & 1 & $0.7 \times 1.4$ & G-E 5 \\
\hline NGC 4472 & 7 & 9 & $0.7 \times 1.4$ & G-E 1 \\
\hline NGC 4621 & 1 & 2 & $0.7 \times 1.4$ & $\mathrm{G}-\mathrm{E} 1$ \\
\hline NGC 7196 & 1 & 1 & $1.9 \times 3.8$ & \\
\hline \multicolumn{5}{|c|}{ Strong LINERS, strong UV turnup } \\
\hline & \multicolumn{2}{|c|}{ G_N4486 } & $\langle 0.8 \times 1.6\rangle$ & \\
\hline NGC 1052 & 4 & 2 & $1.0 \times 1.9$ & \multirow[b]{2}{*}{$\mathrm{G}-\mathrm{E} 1$} \\
\hline NGC 4486 & 5 & 3 & $0.7 \times 1.4$ & \\
\hline \multicolumn{5}{|c|}{ Strong UV turnup } \\
\hline & \multicolumn{2}{|c|}{ G_N4649 } & \multicolumn{2}{|l|}{$\langle 1.4 \times 2.7\rangle$} \\
\hline$\overline{\mathrm{IC}} 4296$ & - & 1 & $2.4 \times 4.8$ & G-E 5 \\
\hline NGC 1399 & 2 & 1 & $1.0 \times 1.9$ & G-E 1 \\
\hline NGC 3894 & - & 1 & $2.1 \times 4.2$ & \\
\hline NGC 4406 & 1 & 1 & $0.7 \times 1.4$ & $\mathrm{G}-\mathrm{E} 1$ \\
\hline NGC 4552 & 3 & 3 & $0.7 \times 1.4$ & $\mathrm{G}-\mathrm{E} 1$ \\
\hline NGC 4649 & 2 & 2 & $0.7 \times 1.4$ & G-E 1 \\
\hline NGC 5266 & - & 1 & $2.0 \times 4.0$ & G-E 5 \\
\hline \multicolumn{5}{|c|}{ Distant galaxies } \\
\hline & \multicolumn{2}{|c|}{ G_N4853 } & \multicolumn{2}{|l|}{$\langle 4.9 \times 9.8\rangle$} \\
\hline NGC 4853 & 2 & 2 & $4.9 \times 9.7$ & \\
\hline NGC 4874 & 1 & - & $4.6 \times 9.2$ & \\
\hline NGC 4889 & 1 & - & $4.2 \times 8.3$ & \\
\hline NGC 6166 & 4 & - & $5.8 \times 11.6$ & \\
\hline NGC 7385 & 1 & - & $5.0 \times 10.0$ & \\
\hline
\end{tabular}

Table Notes. Cols. 2 and 3 give the number of IUE spectra effectively used; Col. 4 gives the projected IUE aperture $\left(10^{\prime \prime} \times 20^{\prime \prime}\right)$ in $\mathrm{kpc} \times \mathrm{kpc}$.

uncertainties were estimated according to the $(S / N)$ ratio in each region. Finally, for the Seyfert groups and control galaxies we measured the Ws and the widths (FWHM) of the most prominent emission lines. Results are given in Table 6.

We selected two strong absorption features in the LWP/R range $\left(\mathrm{Mg} \mathrm{II} \mathrm{I}_{\lambda \lambda 2796,2803}\right.$ and $\left.\mathrm{Mg}_{\lambda 2852}\right)$ and plotted their Ws in Fig. ??, in order to investigate the basic properties of UV lines for galaxies and to compare them to the Ws for star clusters from Paper I. In the upper panel of Fig. ?? we show $W\left(\mathrm{Mg} \mathrm{II} \mathrm{I}_{\lambda \lambda 2796,2803}\right)$ versus $W\left(\mathrm{Mg} \mathrm{I}_{\lambda 2852}\right)$ for the blue stellar population groups and individual galaxies from Table 4. They occupy 


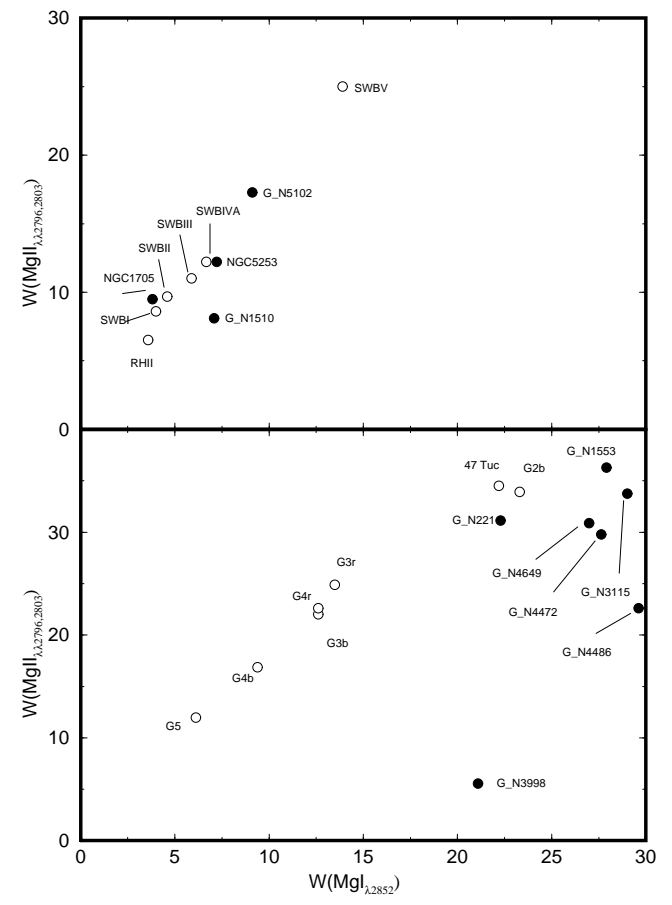

Fig. 3. Equivalent widths of $\mathrm{Mg} \mathrm{I}$ and $\mathrm{Mg}$ II for galaxy and/or groups (filled symbols). Upper panel: blue stellar population groups; lower panel: red stellar population groups. Equivalent widths of star cluster templates (open symbols) are also plotted for comparison (Paper I). Groups of strong LINERs, G_N3998 and G_N4486, have W(Mg II) diluted by emission

similar loci as the blue star clusters SWB I (age $\approx 10 \mathrm{Myr}$ ) to SWBIVA (age $\approx 200 \mathrm{Myr}$ ), and the template RHII which is an average of HII regions in M101, M33, LMC and SMC. The loci of the HII/amorphous galaxies NGC 1705, NGC 5253 and the group G_N1510 suggest that they are not pure HII regions, but contain a stellar population with an age dispersion. In the lower panel, the Ws for the red stellar population galaxy groups are compared to those of the globular cluster templates. For the globular clusters, the sequence from G5 to G $2 \mathrm{~b}$ (and 47 Tuc) increases in metallicity up to $\left[Z / Z_{\odot}\right] \approx-0.5$ (Paper I). As expected, the galaxy groups are close to the metal-rich globular clusters. They present larger Ws in Mg I, indicating higher metallicity, while their Ws are comparable in Mg II. This might be explained by a saturation effect in the galaxies, by the presence of some emission and/or by some interstellar absorption excess on the line of sight to the globular clusters. Notice that the groups G_N3998 and G_N4486, which are strong LINERS, have significantly smaller $W\left(\mathrm{Mg} \mathrm{II}_{\lambda \lambda 2796,2803}\right)$ with respect to the other galaxy groups. This is certainly caused by the contribution of an $\mathrm{Mg} \mathrm{II}_{\lambda \lambda 2796,2803}$ line emission.
Table 3. AGN and blue stellar population spectral groups

\begin{tabular}{|c|c|c|c|c|}
\hline Galaxy & SWP & LWP/R & $\begin{array}{c}\text { slit } \\
(\mathrm{kpc} \times \mathrm{kpc})\end{array}$ & $\begin{array}{c}\text { Spectral } \\
\text { Type }\end{array}$ \\
\hline \multicolumn{5}{|c|}{ Seyfert 1's - bluer ones } \\
\hline & \multicolumn{2}{|c|}{ G_Mk 279} & $\langle 6.0 \times 11.9\rangle$ & \\
\hline$\overline{\mathrm{Mk} 9}$ & 4 & 3 & $7.5 \times 15.1$ & \\
\hline $\mathrm{Mk} 279$ & 25 & 13 & $5.7 \times 11.4$ & \\
\hline $\mathrm{Mk} 290$ & 5 & 7 & $5.6 \times 11.3$ & \\
\hline Mk 352 & 2 & 1 & $2.9 \times 5.8$ & \\
\hline Mk 1018 & 6 & 1 & $7.9 \times 15.7$ & \\
\hline \multirow[t]{3}{*}{ Mk 1506} & 40 & 19 & $6.2 \times 12.4$ & \\
\hline & \multicolumn{3}{|c|}{ Seyfert 1's - redder ones } & \\
\hline & \multicolumn{2}{|c|}{ G_N3516 } & $\langle 3.9 \times 7.7\rangle$ & \\
\hline ESO 252-G18 & 2 & 1 & $6.6 \times 13.2$ & \\
\hline $\mathrm{MCG}+08-31-0041$ & 2 & 1 & $4.7 \times 9.3$ & \\
\hline Mk 705 & 1 & - & $5.6 \times 11.1$ & \\
\hline NGC 3516 & 35 & 23 & $1.7 \times 3.4$ & \\
\hline \multirow{3}{*}{ NGC 5273} & 2 & 1 & $0.7 \times 1.4$ & \\
\hline & \multicolumn{3}{|c|}{ Seyfert2's } & \\
\hline & \multicolumn{2}{|c|}{ G_Mk 573} & $\langle 2.6 \times 5.1\rangle$ & \\
\hline$\overline{\mathrm{ESO}} 434-\mathrm{G} 40$ & 1 & - & $1.6 \times 3.2$ & \\
\hline IC 450 & 2 & 3 & $3.5 \times 7.1$ & \\
\hline IC 5063 & 1 & 1 & $2.2 \times 4.4$ & \\
\hline Mk 198 & 1 & 1 & $4.6 \times 9.2$ & \\
\hline Mk 573 & 2 & 1 & $3.4 \times 6.7$ & \\
\hline NGC 1106 & 1 & - & $2.7 \times 5.5$ & \\
\hline NGC 2110 & 1 & 1 & $1.5 \times 3.0$ & \\
\hline NGC 5283 & 1 & 1 & $2.3 \times 4.7$ & \\
\hline \multirow{3}{*}{ NGC 7465} & 1 & - & $1.3 \times 2.6$ & \\
\hline & \multicolumn{3}{|c|}{ HII/amorphous galaxies } & \\
\hline & & 1510 & $\langle 1.5 \times 3.0\rangle$ & \\
\hline$\overline{\mathrm{Mk} 49}$ & 1 & - & $1.1 \times 2.2$ & \\
\hline Mk 960 & 1 & 1 & $3.5 \times 7.0$ & \\
\hline NGC 1510 & 2 & 1 & $0.6 \times 1.3$ & \\
\hline NGC 3125 & 2 & 1 & $0.7 \times 1.4$ & \\
\hline \multirow[t]{3}{*}{ NGC 4385} & 1 & 1 & $1.5 \times 3.0$ & \\
\hline & \multicolumn{3}{|c|}{ Ageing bursts } & \\
\hline & \multicolumn{2}{|c|}{ G_N5102 } & $\langle 0.3 \times 0.6\rangle$ & \\
\hline$\overline{\mathrm{NGC} 185}$ & - & 1 & $0.03 \times 0.06$ & \\
\hline NGC 205 & 4 & 7 & $0.03 \times 0.06$ & \\
\hline NGC 3156 & 2 & - & $0.7 \times 1.5$ & \\
\hline \multirow[t]{2}{*}{ NGC 5102} & 5 & 3 & $0.3 \times 0.6$ & $\mathrm{G}-\mathrm{E} 8$ \\
\hline & \multicolumn{2}{|c|}{ Individual objects } & $\langle 0.8 \times 1.6\rangle$ & \\
\hline$\overline{\mathrm{IC}} 4329 \mathrm{~A}$ & 1 & 2 & $3.1 \times 6.2$ & \\
\hline NGC 404 & 3 & 2 & $0.2 \times 0.4$ & \\
\hline NGC 1326 & 1 & 1 & $0.9 \times 1.9$ & \\
\hline NGC 1705 & 10 & 14 & $0.4 \times 1.8$ & \\
\hline NGC 1947 & 1 & - & $0.7 \times 1.5$ & \\
\hline NGC 2784 & 1 & 1 & $0.4 \times 0.9$ & \\
\hline NGC 5128 & 13 & 10 & $0.3 \times 0.6$ & \\
\hline \multirow[t]{2}{*}{ NGC 5253} & 6 & 6 & $0.3 \times 0.6$ & $\mathrm{G}-\mathrm{S} 7$ \\
\hline & \multicolumn{2}{|c|}{ Control galaxies } & $\langle 0.7 \times 1.4\rangle$ & \\
\hline$\overline{\text { NGC } 1068}$ & 27 & 12 & $0.7 \times 1.5$ & \\
\hline NGC 4151 & 326 & 247 & $0.6 \times 1.3$ & \\
\hline NGC 5236 & 2 & 7 & $0.3 \times 1.6$ & \\
\hline NGC 7213 & 8 & 5 & $1.2 \times 2.3$ & \\
\hline
\end{tabular}

\section{Discussion}

\subsection{Normal galaxies}

The red stellar population groups, in particular the analyses of the UV turnup, are discussed in a separate paper (Bica et al. 1995). Here we simply point out that the UV turnup exhibits possible absorption features, at least as detected through the IUE aperture (Fig. ??). However, their reality still remains to be confirmed, owing to the presence of camera artifacts in long-exposure spectra (Crenshaw et al. 1990). On the other hand, we call 
Table 4. Equivalent width measurements of absorption lines in red and blue stellar population groups
Table 5. Continuum measurements $-C_{\lambda} / C_{2646}$ 
attention to Fig. 8a of Paper I, in which we averaged all red stellar population star clusters in the IUE database, and the resulting very high $(S / N)$ spectrum presents absorption features which can be recognised in the galaxy templates. Besides, the different redshift corrections tend to minimise the effects of camera artifacts. In what follows, we use some red groups as templates in some spectral syntheses.

\subsubsection{Blue stellar populations}

We compare in Fig. ?? the spectrum of the group G_N1510 (average of HII and amorphous galaxies) to that of NGC 5253. As expected, the spectra are very similar. Emission lines are not characteristic features of the UV spectrum of H II galaxies. We also display in Fig. ?? the HII region template from Paper I (including SMC, LMC, M 33 and M $101 \mathrm{HII}$ regions), the star cluster templates SWB I (age $\approx 10 \mathrm{Myr}$ ) and SWB II (age $\approx 25 \mathrm{Myr}$ ). The HII region template does not show either conspicuous emission lines (Paper I). Thus, the presence of strong emission lines in the UV spectra of galaxies, e.g. $\mathrm{Mg} \mathrm{II}_{\lambda \lambda 2796,2803}$, such as in those of Seyfert nuclei (Fig. ??) is not related to conventional star-formation, indicating the presence of activity. The different slopes of the HII region template and the HII galaxy spectra might be explained by a larger internal reddening in NGC 5253 and G_N1510. If this is the case, the extinction law would necessarily be similar to that of the SMC because of the absence of the $\lambda 2200 \AA$ absorption feature in the H II galaxies. Alternatively, the slope could also be changed by a combination of the $\mathrm{HII}$ template and other recent star formation templates, e.g. SWB II. Such galaxies would have been forming stars over the last $\approx 50 \mathrm{Myr}$.

We compare in Fig. ?? the spectrum of the group G_N5102 to those of the star cluster templates SWB III (age $\approx 75 \mathrm{Myr}$ ), SWB IVA (age $\approx 200 \mathrm{Myr}$ ) and SWBV (age $\approx 1.2 \mathrm{Gyr}$ ). Two members of this galaxy group, NGC5102 and the M31 companion NGC 205, were population-synthesized in the visible and near-IR ranges using a star cluster base in Bica (1988, NGC 5102 therein referred to as template E 8) and Bica et al. (1990), respectively. It was concluded that these galaxies had a strong burst of star formation at ages $t \approx 100-500 \mathrm{Myr}$. In the UV (Fig. ??) we find that the cluster template SWB IVA $(\approx 200 \mathrm{Myr})$ must be a major contributor to the G_N5102 group, and therefore we confirm the presence of a strong burst in the age range $100-500 \mathrm{Myr}$.

\subsubsection{Atypical spectra}

Some spectra in the sample did not fit the group classifications. In the following we discuss their properties.

NGC 1326. The spectrum of NGC 1326 (Fig. ??) exhibits on its short wavelength range a slope and spectral

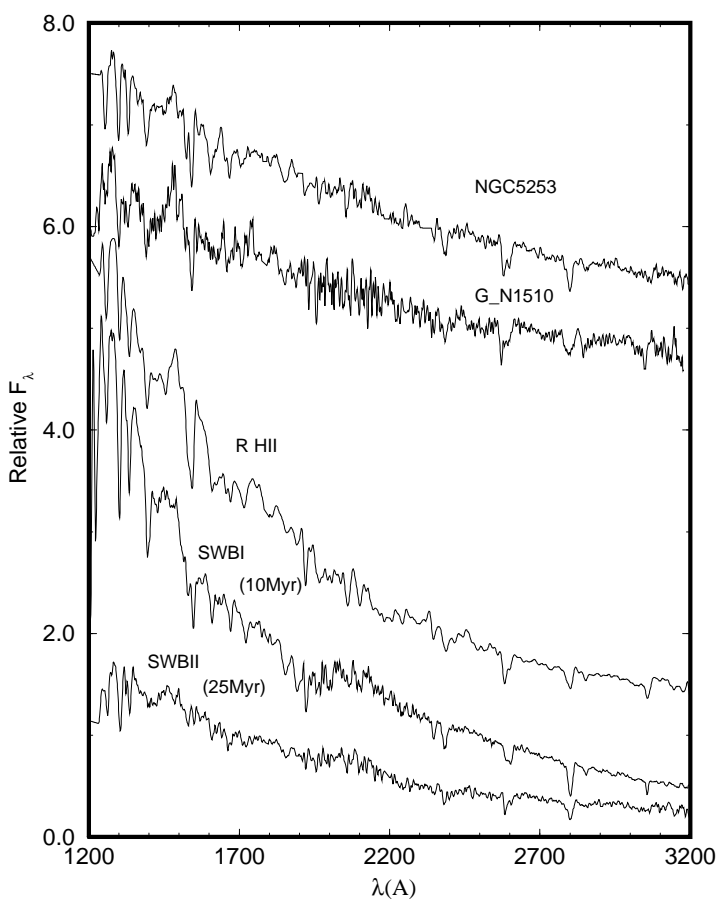

Fig. 4. The spectrum of the group G_N1510 is compared to that of NGC 5253 and to those of H II region and star cluster templates of different ages. Fluxes are in $F_{\lambda}$ units, normalised at $\lambda 2646 \AA$. Constants have been added to the spectra for clarity purposes, except for the bottom one

features similar to those characterizing the nuclear starburst in NGC 5236, whereas at $\lambda>2500 \AA$ the flux increases as for a red stellar population (groups G_N1553 and G_N3115 in Fig. ??). We have undertaken a first level synthesis by adding these templates with different relative proportions and conclude that their proportions at the normalization wavelength $\lambda 2646 \AA$ are $85 \%$ of a burst and $15 \%$ of the red population (see Fig. ??).

NGC 404. We compare in Fig. ?? the spectrum of the nearby dwarf galaxy NGC 404 with that of the red stellar population group G_N221. The stellar population synthesis in the visible/near-IR of NGC 404, which is a strong LINER as well (Schmidt et al. 1990), revealed a bulge population hardly attaining solar metallicity with evidence of young and intermediate age components. These young components are seen indeed as an excess flux in the UV as compared to the red group G_N221. They also have an impact on the relative intensities of strong absorption lines such as Fe II, MgII and MgI (Fig. ??).

NGC 2784. The IUE spectrum of the galaxy NGC 2784 has a rather low $(S / N)$ ratio, allowing one only to infer a slope, intermediate between nuclear starburst spectra like NGC 5236 and the group G_N5102 (Figs. ?? and ??, 
Table 6. Emission-line parameters

\begin{tabular}{|c|c|c|c|c|c|c|c|c|c|c|}
\hline \multirow[b]{2}{*}{ Galaxy } & \multicolumn{2}{|c|}{$\operatorname{SiIII}_{\lambda 1400}$} & \multicolumn{2}{|c|}{$\mathrm{CIV}_{\lambda 1549}$} & \multicolumn{2}{|c|}{$\mathrm{HeII}_{\lambda 1640}$} & \multicolumn{2}{|c|}{ CIII $]_{\lambda 1909}$} & \multicolumn{2}{|c|}{$\mathrm{MgII}_{\lambda \lambda 2798,2803}$} \\
\hline & $\begin{array}{l}\mathrm{W} \\
(\AA)\end{array}$ & $\begin{array}{c}\text { FWHM } \\
(\AA)\end{array}$ & $\begin{array}{l}\mathrm{W} \\
(\AA)\end{array}$ & $\begin{array}{c}\text { FWHM } \\
(\AA)\end{array}$ & $\begin{array}{l}\mathrm{W} \\
(\AA)\end{array}$ & $\begin{array}{c}\text { FWHM } \\
(\AA)\end{array}$ & $\begin{array}{l}\mathrm{W} \\
(\AA)\end{array}$ & $\begin{array}{c}\text { FWHM } \\
(\AA)\end{array}$ & $\begin{array}{l}\mathrm{W} \\
(\AA)\end{array}$ & $\begin{array}{c}\text { FWHM } \\
(\AA)\end{array}$ \\
\hline$\overline{\text { G_Mk279 }}$ & 13.9 & 27.2 & 78.4 & 28.5 & 7.3 & 24.5 & 22.4 & 32.6 & 34.8 & 44.1 \\
\hline G_N3516 & 8.2 & 21.3 & 93.1 & 36.7 & 10.2 & 18.0 & 41.1 & 39.3 & 31.4 & 45.3 \\
\hline G_Mk573 & 67.8 & 25.0 & 139.2 & 15.8 & 69.3 & 10.7 & 79.7 & 7.4 & - & - \\
\hline NGC4151 & 10.7 & 14.0 & 177.7 & 24.8 & 24.3 & 10.5 & 63.7 & 16.0 & 76.6 & 41.9 \\
\hline NGC7213 & 25.2 & 36.4 & 120.8 & 32.6 & 34.3 & 33.5 & 54.9 & 26.1 & 84.7 & 34.9 \\
\hline NGC1068 & 14.3 & 14.3 & 60.8 & 12.3 & 27.5 & 12.4 & 24.3 & 15.1 & 14.6 & 18.4 \\
\hline
\end{tabular}

Table Notes. Equivalent width and FWHM of the most prominent emission lines present in the Seyfert groups; control galaxies are used for comparison.

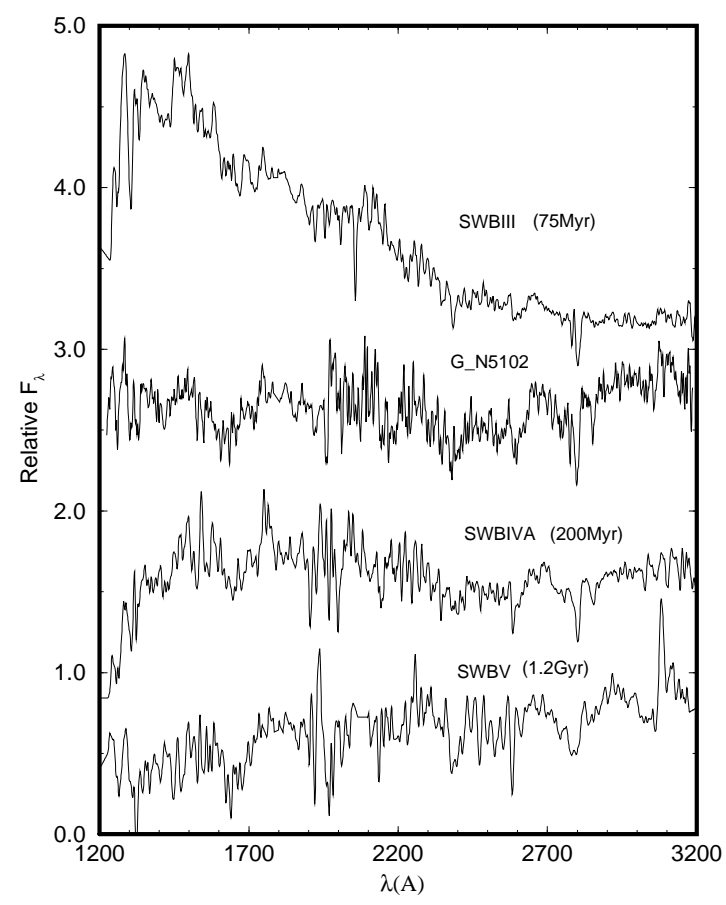

Fig. 5. The spectrum of the blue population group G_N5102 is compared to those of star cluster templates at different ages. Fluxes are in $F_{\lambda}$ units, normalised at $\lambda 2646 \AA$. Constants have been added to the spectra for clarity purposes, except for the bottom one

respectively). Indeed, this $\mathrm{S} 0$ galaxy, also known as AM 0910-235, may have developed a nuclear starburst since it shows signs of interaction with its dwarf companions (Arp \& Madore 1987). Visible and near-IR spectra, as well as more exposed UV spectra, are necessary to further check this interesting possibility.

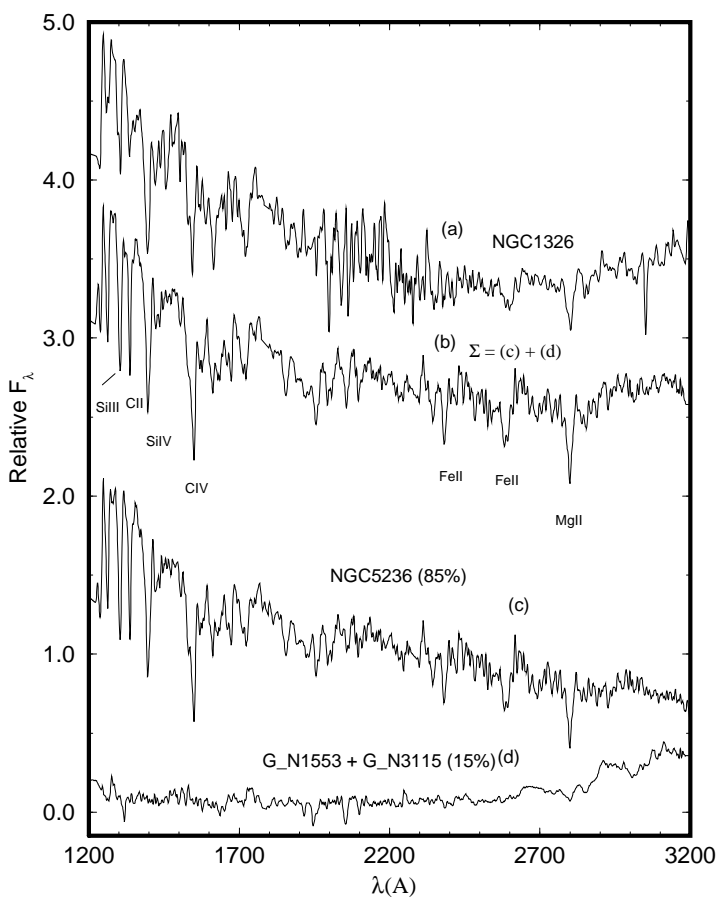

Fig. 6. a) Spectrum of NGC 1326; b) synthesis; c) burst and d) red stellar-population components. For a) and b) fluxes are in $F_{\lambda}$ units normalised at $\lambda 2646 \AA$, while constants have been added to the spectra for clarity purposes. In c) and $\mathbf{d}$ ), actual $F_{\lambda}$ proportions at $\lambda 2646 \AA$ are shown

\subsection{Seyfert galaxies}

We obtained two groups of Seyfert 1 galaxies (Fig. ??): the spectrum of G_Mk279 is notably bluer than that of G_N3516. Otherwise, the equivalent widths and FWHM of the emission lines (Table 6) do not show systematic differences. In principle, three possibilities might account for the continuum difference between the two groups: i) a reddening difference; ii) that the galaxies in the two groups would be in a different average activity state; and 


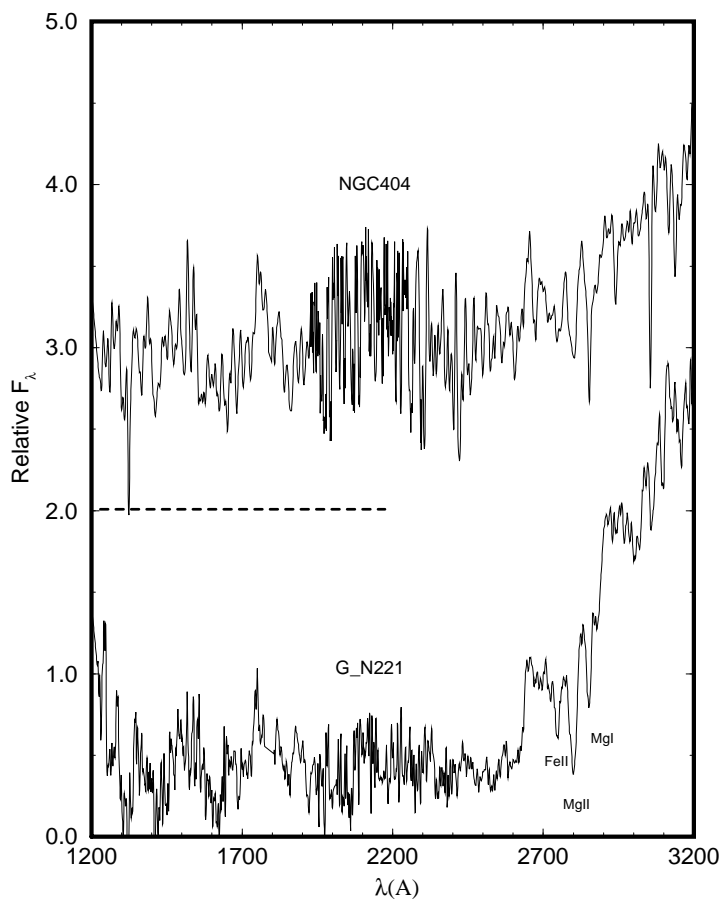

Fig. 7. No UV turnup is detected in the spectrum of the red stellar population group G_N221. The spectrum of the nearby dwarf galaxy NGC 404 shows evidence of young and intermediate age components. Fluxes are in $F_{\lambda}$ units, normalised at $\lambda 2646 \AA$. A constant has been added to the upper spectrum for clarity purposes; the dashed line indicates the true zero level for NGC 404

iii) that the G_Mk279 group contains additionally a starburst component. We applied several tests in order to constrain these possibilities. If differential internal reddening is responsible for the effect, the reddening law should not have a strong $\lambda 2200 \AA$ bump. This can be seen in Fig. ??, where the SMC extinction law (Prévot et al. 1984) with $E(B-V)=0.10$, when applied to the G_N3516 spectrum, reproduces quite well that of G_Mk279. On the other hand, Seaton's (1979) law with $E(B-V)=0.15$ already overestimates the $\lambda 2200 \AA$ correction and, at the same time, underestimates the far-UV flux.

Assuming that the emission lines are comparable in the two groups of Seyfert 1 galaxies, we normalised the spectra to the $\mathrm{Mg} \mathrm{II} \mathrm{I}_{\lambda \lambda 2796,2803}$ line intensity and then obtained the difference spectrum G_Mk279 - G_N3516. In Fig. ?? we compare the difference spectrum to that of the typical nuclear starburst NGC5236, and also to the power-law $F_{\lambda} \propto \lambda^{-1.8}$. The power-law fits well the continuum of the difference spectrum, and so does the starburst continuum. However, the starburst absorption lines are not conclusively present in the difference spectrum. On the other hand, both a power-law or a starburst continuum with such a steep slope would cause a considerable dilution of

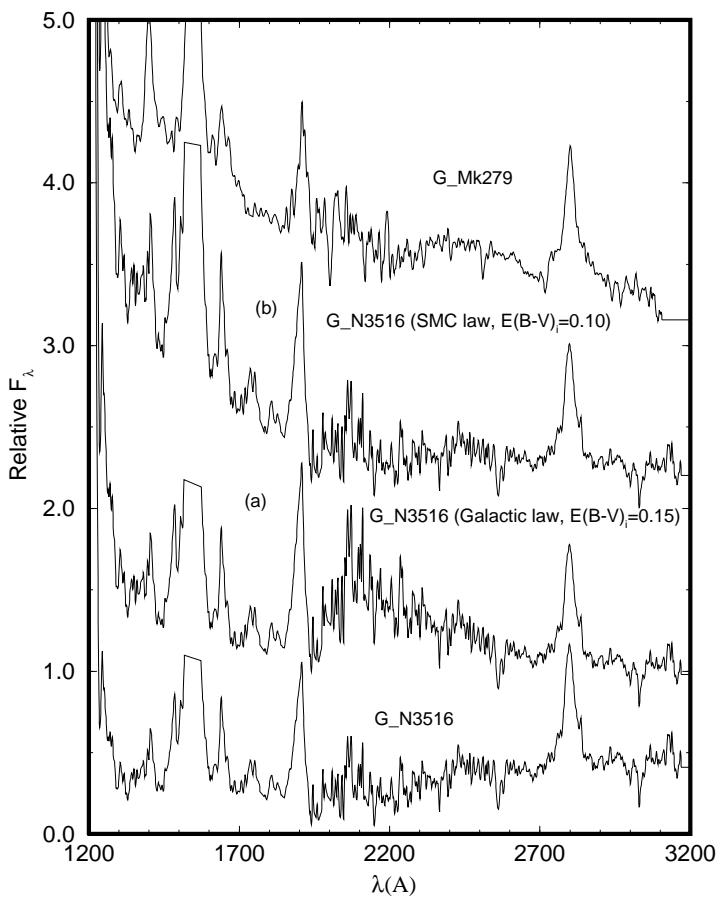

Fig. 8. The redder Seyfert 1 group G_N3516 has been reddening corrected using a) a Galactic extinction law with $E(B-V)=0.15$, and b) the SMC law with $E(B-V)=0.10$. As compared to the bluer Seyfert 1 group G_Mk279, the Galactic law underestimates the far-UV flux and overestimates the $\lambda 2200 \AA$ correction, whereas the SMC law produces a very similar spectrum. Fluxes are in $F_{\lambda}$ units, normalised at $\lambda 2646 \AA$. Constants have been added to the spectra for clarity purposes, except for the bottom one. The strong C IV emission has been clipped for clarity

the equivalent widths of the emission lines of G_Mk279 with respect to those of $G_{-} N 3516$, which is not the case (Table 6).

Therefore, we conclude that internal reddening is a more likely cause of the continuum difference between the two Seyfert 1 groups. Within the scenario of the Unified Model of AGNs (Antonucci 1993), Seyfert 2 galaxies would be Seyfert 1s but with their BLR and central source hidden by a dusty torus. Within this frame, the active nuclei of galaxies in the group G_N3516 could be interpreted as seen at lines of sight which cross a larger amount of the dusty torus than in the case of the group G_Mk279. Would this be the case, our results from Fig. ?? imply that the extinction law in the dusty torus does not have a conspicuous $\lambda 2200 \AA$ bump, and resembles that of the SMC.

We draw attention to the fact that in the group of the bluest Seyfert 1s (G_Mk279), the galaxy Mk 1506 exhibits a conspicuous absorption around $\lambda 2200 \AA$ which denotes the presence of significant differential internal reddening with respect to the other Seyfert 1s in the group. Before 


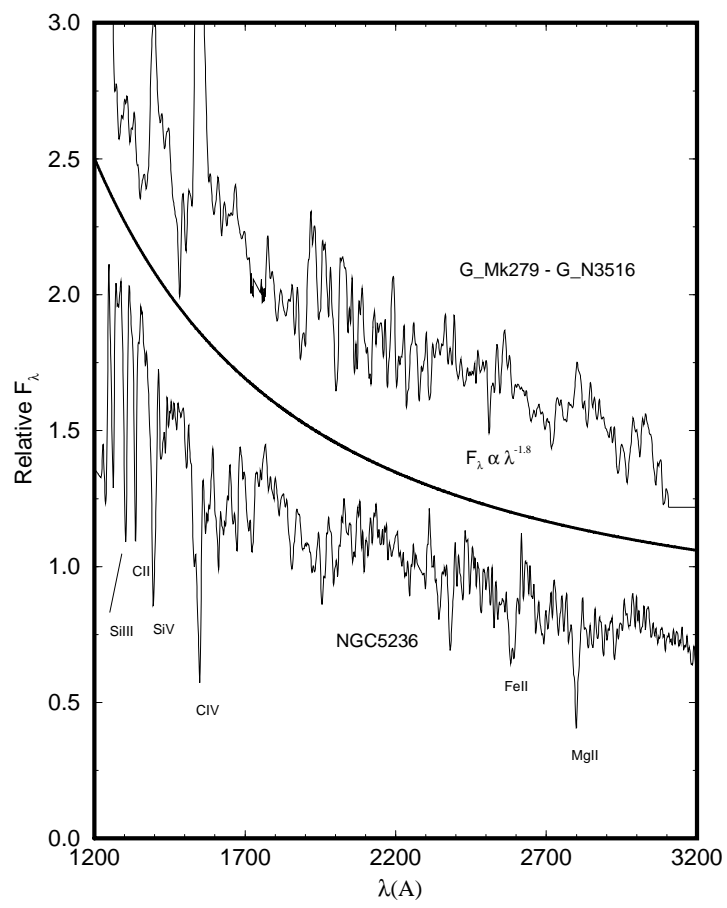

Fig. 9. The difference spectrum between the bluer (G_Mk279) and redder (G_N3516) Seyfert 1 groups is compared to a power-law continuum (thick solid line) and to the nuclear starburst NGC 5236. Fluxes are in $F_{\lambda}$ units, normalised at $\lambda 2646 \AA$. Constants have been added to the spectra for clarity purposes, except for the bottom one

including Mk 1506 in the G_Mk279 group we corrected its spectrum for internal reddening effects. We considered the Galactic reddening law (Seaton 1979) which presents a bump at $\lambda \approx 2200 \AA, R=3.1$ and derived $E(B-V)_{i}=$ 0.20 (Fig. ??).

In Fig. ?? we compare the SWP region of the Seyfert 2 group G_Mk573 with that of the Seyfert 2 prototype NGC 1068. It is worth noting that individual IUE spectra building up the G_Mk573 group are noisy and do not release much information. We decided that they were Seyfert 2 galaxies relying on their visible spectra counterpart (references in Sects. 2 and 3). The resulting UV spectrum of the group confirms the Seyfert 2 nature of its members because of its similarity to the spectrum of NGC 1068. The FWHM for the ensemble of the emission lines (Table 6) reinforces this conclusion. As to the equivalent widths, those in the group G_Mk573 are systematically larger than those of NGC 1068 (Table 6), suggesting a higher excitation and/or less contamination by the stellar continuum component in the former.

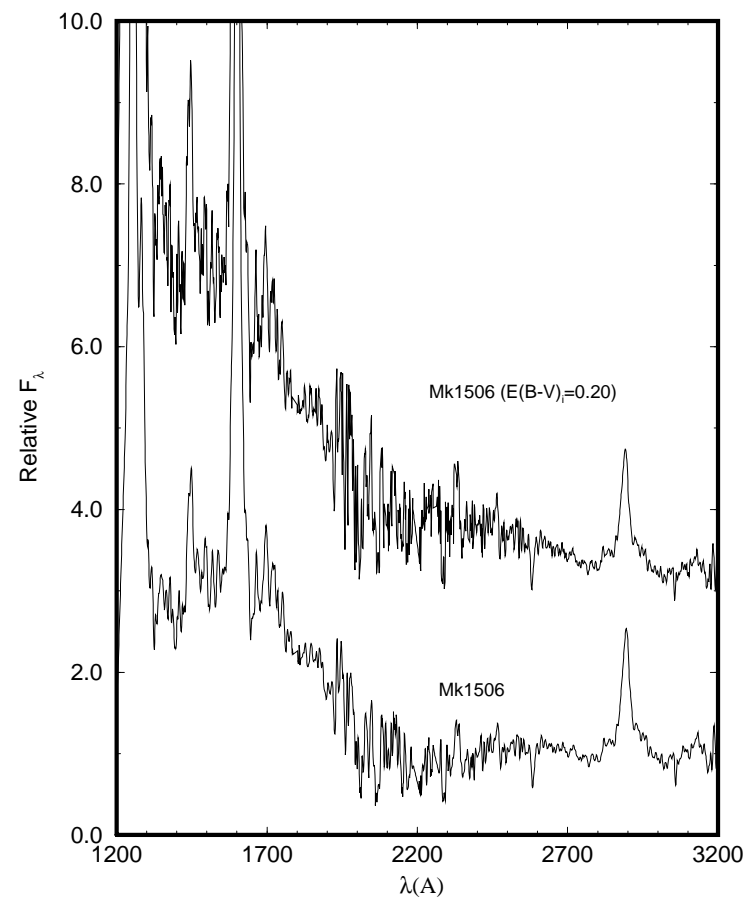

Fig. 10. The Seyfert 1 galaxy Mk 1506 has been reddening corrected using a Galactic law with $E(B-V)_{i}=0.20$, which successfully cancels out the $\lambda 2200 \AA$ absorption. Fluxes are in $F_{\lambda}$ units normalised at $\lambda 2646 \AA$. A constant was added to the upper spectrum for clarity purposes

\subsection{Internally reddened galaxies}

The galaxies NGC5128, NGC 1947 and the Seyfert 1 IC $4329 \mathrm{~A}$ are known to have their nuclei reddened by strong dust lanes, as derived from optical spectra and imaging (see RC3 and references therein cited).

\subsubsection{NGC 5128}

In the averaging procedure for NGC5128 we have excluded the spectra SWP 1799 and LWR 1681 which appear to be related to a Galactic field star of spectral type A, and SWP 14164 and LWR 10749 which correspond to an H II region in the disk of NGC 5128 (Rosa et al. 1984). The remaining 13 SWP and $10 \mathrm{LWP} / \mathrm{R}$ spectra all look similar within the available $(S / N)$ ratios, and their average is shown in Fig. ??. We also display in Fig. ?? the SWP spectrum of NGC 1947 which, within the available $(S / N)$ ratio, is compatible with that of NGC 5128. Compared to a typical red stellar population in early-type galaxies, e.g. the average of the groups G_N1553 and G_N3115 (Fig. ??), NGC 5128 presents a steeper slope at $\lambda<2500 \AA$ and significant differences in the intensity of the absorption features $\mathrm{MnII}+\mathrm{FeII}_{\lambda 2577,2586}, \mathrm{Mg} \mathrm{II}_{\lambda \lambda 2796,2803}, \mathrm{Mg} \mathrm{I}_{\lambda 2852}$ and $\mathrm{Fe} \mathrm{I}$ lines in the range $\lambda \lambda 3008-3059 \AA$. 


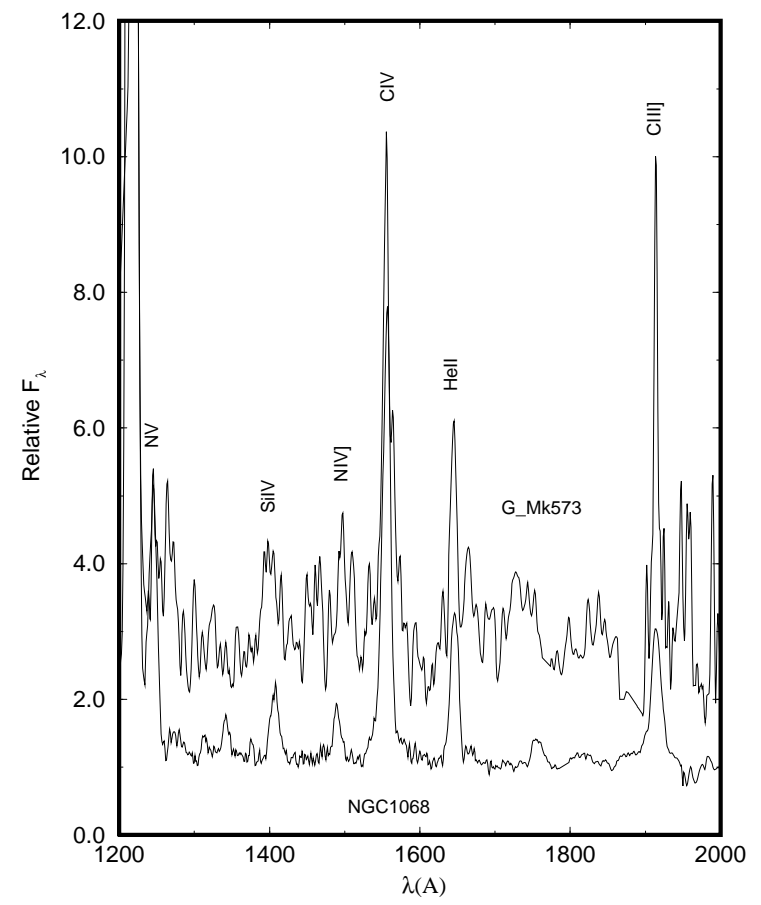

Fig. 11. The SWP region of the Seyfert 2 group G_Mk573 is compared to that of NGC 1068; emission lines in G_Mk573 have similar width and relative intensities as those of NGC 1068. Fluxes are in $F_{\lambda}$ units, normalised at $\lambda 2646 \AA$. A constant was added to the upper spectrum for clarity purposes

In order to interpret the spectrum of NGC 5128, we assumed a disk population which can be represented by the average of the star cluster templates SWBI (age $\approx 10 \mathrm{Myr}$ ), SWBIVA (age $\approx 200 \mathrm{Myr}$ ) and SWBV (LMC+SMC, age $\approx 1-2 \mathrm{Gyr}$ ), and a bulge population which can be represented by the average of the galaxy groups G_N1553 and G_N3115. The relative proportions of disk and bulge components at $\lambda 2646 \AA$ were found to be respectively, 0.9 and 0.1 , a mixture which reproduces also reasonably well the absorption lines in NGC 5128 (Fig. ??). The resulting continuum ( $\Sigma$ in Fig. ??) requires some additional reddening in order to reproduce that of NGC 5128. Galactic and LMC reddening laws (Seaton 1979; Fitzpatrick 1986) are not suitable in this case, as they would produce too strong an absorption at $\lambda 2200 \AA$. On the other hand, the SMC law (Prévot et al. 1984) with $E(B-V)=0.30$ (assuming $R=3.1$ ) provides a good fit. This is another case of an external galaxy other than the Magellanic Clouds where the properties of the reddening law in the UV can be derived. Recently, Kinney et al. (1994) found that the $\lambda 2200 \AA$ bump of the reddening law is not present in another galaxy (NGC 7552). The large proportion of the disk component relative to that of the bulge suggests that the nuclear regions of NGC5128 are not contributing much to the integrated

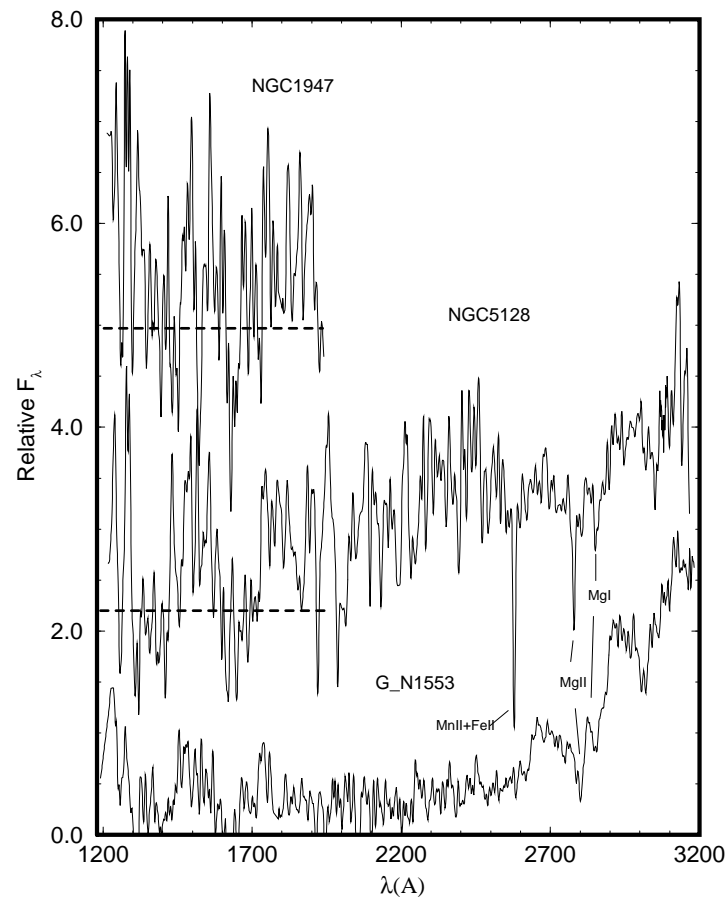

Fig. 12. Internally reddened galaxies NGC5128 and NGC 1947 compared to the red stellar population group G_N1553. Flux in $F_{\lambda}$ units, normalised at $\lambda 2646 \AA$. Constants have been added to the spectra for clarity purposes, except for the bottom one; the true zero-levels for the upper spectra are shown as dashed lines

spectrum observed through the IUE aperture. The optical depth through the dust lane appears to be so large for the nucleus in the far-UV that only templates of the external shells in the bulge and of the disk would be relevant. The pronounced depth and narrow width of the $\mathrm{Mn} \mathrm{II}_{\lambda 2577}$ and/or Fe $\mathrm{II}_{\lambda 2586}$, and also of the $\mathrm{Mg} \mathrm{II}_{\lambda \lambda 2796,2803}$ features suggest that some interstellar contribution occurs. This interstellar component might have arisen either in the disk of NGC 5128 or in our Galaxy, because of the low radial velocity and low Galactic latitude of NGC 5128.

\subsubsection{IC $4329 \mathrm{~A}$}

The Seyfert 1 galaxy IC 4329A has a very reddened visible spectrum which becomes comparable to that of the almost face-on galaxy NGC 3783 after an internal reddening correction with $E(B-V)_{i}=0.79$ has been applied (Bica \& Alloin 1986). We show in the lower panel of Fig. ?? the IUE spectrum of IC 4329A (foreground reddening corrected, Sect. 2) where a conspicuous absorption around $\lambda 2200 \AA$ indicates that the reddening law associated to the disk of this galaxy has a UV bump. Here we simply consider and apply the same excess $E(B-V)_{i}$ as for the visible and the Galactic reddening law (Seaton 1979): in the 


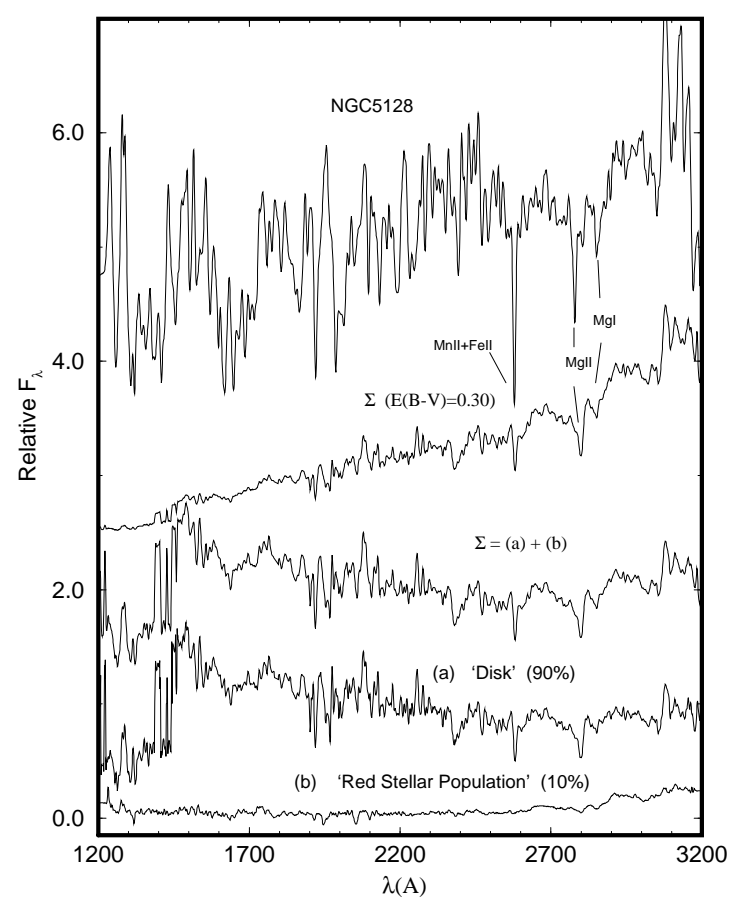

Fig. 13. Synthesis of NGC 5128. Components are: $10 \%$ of a red stellar population and $90 \%$ of a disk population. The resulting spectrum was reddened using the SMC law with $E(B-V)=0.30$. Flux in $F_{\lambda}$ units, normalised at $\lambda 2646 \AA$. Constants have been added to the spectra for clarity purposes. In a) and b) actual $F_{\lambda}$ proportions at $\lambda 2646 \AA$ are shown

upper panel of Fig. ??, we compare the resulting spectrum to that of our control Seyfert 1 in the UV (NGC 4151). Indeed the $\lambda 2200 \AA$ absorption is basically cancelled out. However, no prominent emission lines are detected in the corrected spectrum of IC 4329A. In the SWP region the noise level is large indeed, but still, even the strong emission line of C IV usual in AGNs does not show up. In the $\mathrm{LWP} / \mathrm{R}$ region, the $\mathrm{Mg} \mathrm{II}_{\lambda \lambda 2796,2803}$ line emission is not detected either in IC 4329A. We conclude that the optical depth is so large for the AGN in the UV that its emission lines and continuum are hardly detectable.

\subsection{Strong LINERs}

The groups G_N3998 (formed by NGC 3998 and NGC 4278) and G_N4486 (NGC 4486 = M 87 and NGC 1052), host well-known and strong LINERs (see references in Sects. 2 and 3). The equivalent width analysis (Sect. 4) has shown that $\mathrm{Mg} \mathrm{II}_{\lambda \lambda 2796,2803}$ in emission is probably causing the decrease of the absorption intensity in the corresponding window. The stellar population in G_N4486 is very similar to that of the group G_N4649 (Fig. ??) which has a strong UV turnup. The group G_N3998 has a red stellar population with far-

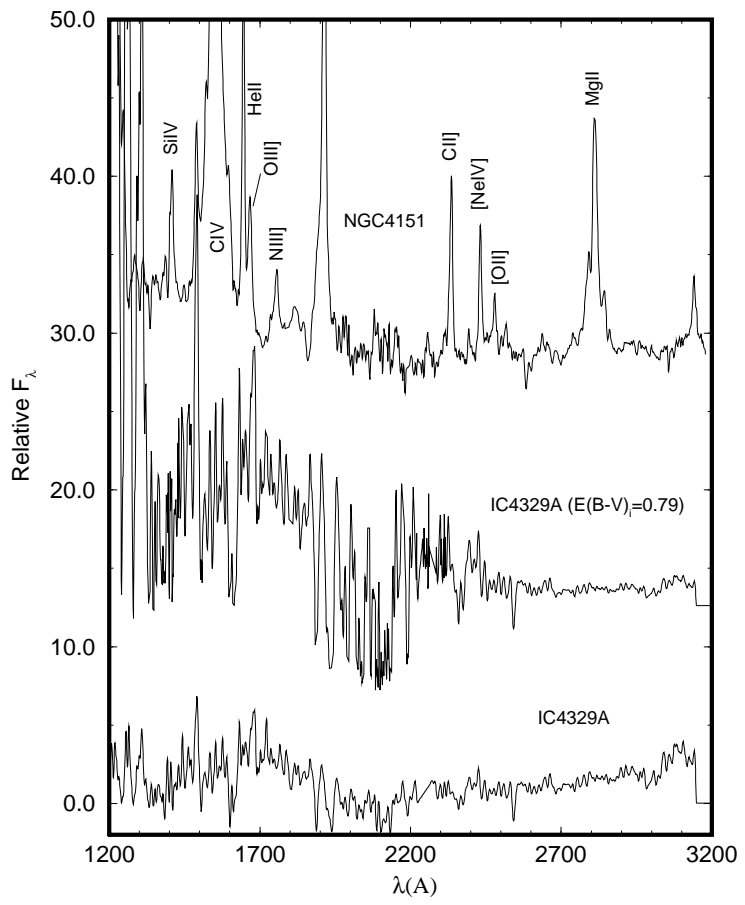

Fig. 14. The Seyfert 1 galaxy IC 4329A has been reddening corrected using the Galactic law with $E(B-V)=0.79$. The resulting spectrum is compared to that of NGC 4151; a roughly similar slope is found. Flux in $F_{\lambda}$ units, normalised at $\lambda 2646 \AA$. Constants have been added to the spectra for clarity purposes, except for the bottom one

UV flux intermediate between the groups G_N4472 and G_N3115 (Fig. ??). It seems however to be disturbed by emissions such as Mg II, C IV and C III]. We investigate in Figs. ?? and ?? the possibility of a mini-Seyfert nucleus being present in both groups. Indeed, a contribution like NGC 7213 (a Seyfert 1/LINER galaxy nested in the bulge of an Sa galaxy) amounting to $20 \%$ of the flux at $\lambda 2646 \AA$, when added to the spectrum of the group G_N4472, reproduces well the overall spectrum of G_N3998 (Fig. ??). A similar mixture can be performed for the G_N4486 group with an even smaller contribution from NGC 7213 added to the group G_N4649 (Fig. ??). In both the G_N3998 and G_N4649 groups, the presence of the Seyfert nucleus does not account for the UV turnup. In the case of G_N4486, this component cannot contribute more than $10 \%$ at $\lambda 2646 \AA$.

\section{Concluding remarks}

We have gathered the UV spectra of the early-type galaxies (ellipticals and S 0's) stored in the IUE database. We grouped the objects showing similar spectral characteristics from the UV to the visible/near-IR ranges. This procedure provided average spectra with improved $(S / N)$ ratio, 


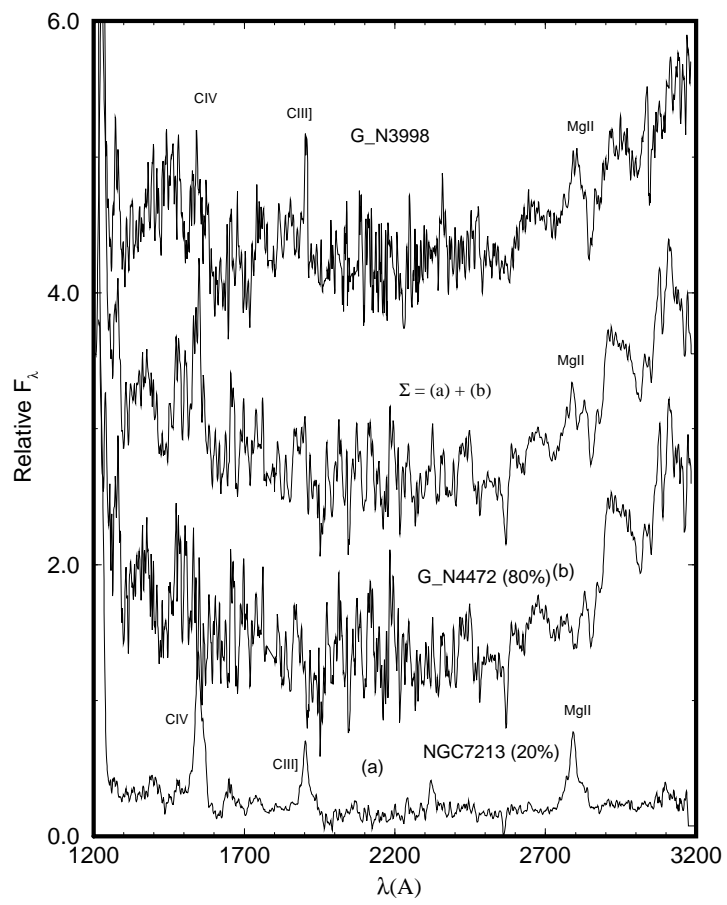

Fig. 15. The spectrum of the strong LINER group G_N3998 can be reproduced by combining those of the Seyfert 1/LINER NGC 7213 and the galaxy group G_N4472; proportions are, respectively, $20 \%$ and $80 \%$ of the flux at $\lambda 2646 \AA$. Flux in $F_{\lambda}$ units, normalised at $\lambda 2646 \AA$. Constants have been added to the spectra for clarity purposes, except for the bottom one

giving access to more reliable information about spectral features. The main results of this work are the following:

i) We obtained several spectral groups characterised by blue and red stellar populations, as well as others containing AGN;

ii) the UV turnup possibly presents absorption features, at least as detected through the IUE aperture, although camera artifacts may still be affecting this region;

iii) using star cluster templates of different ages, it was possible to infer the age distribution in the galaxy spectra dominated by blue stellar populations;

iv) two distinct Seyfert 1 galaxy groups were obtained: one (G_Mk279) is considerably bluer than the other (G_N3516); this difference in continuum slope can be fully accounted for by internal reddening only if we assume an SMC-like extinction law, i.e. without the $\lambda 2200 \AA$ bump;

v) the galaxies classified as Seyfert 2 in the visible/near-IR ranges form the group G_Mk573. Their combined UV spectrum turns out to be similar to the spectrum of the Seyfert 2 prototype NGC 1068, thus confirming the group members as Seyfert 2's in this wavelength range as well;

vi) we carried out a simple population synthesis of the spectrum of the reddened galaxy NGC5128. A good re-

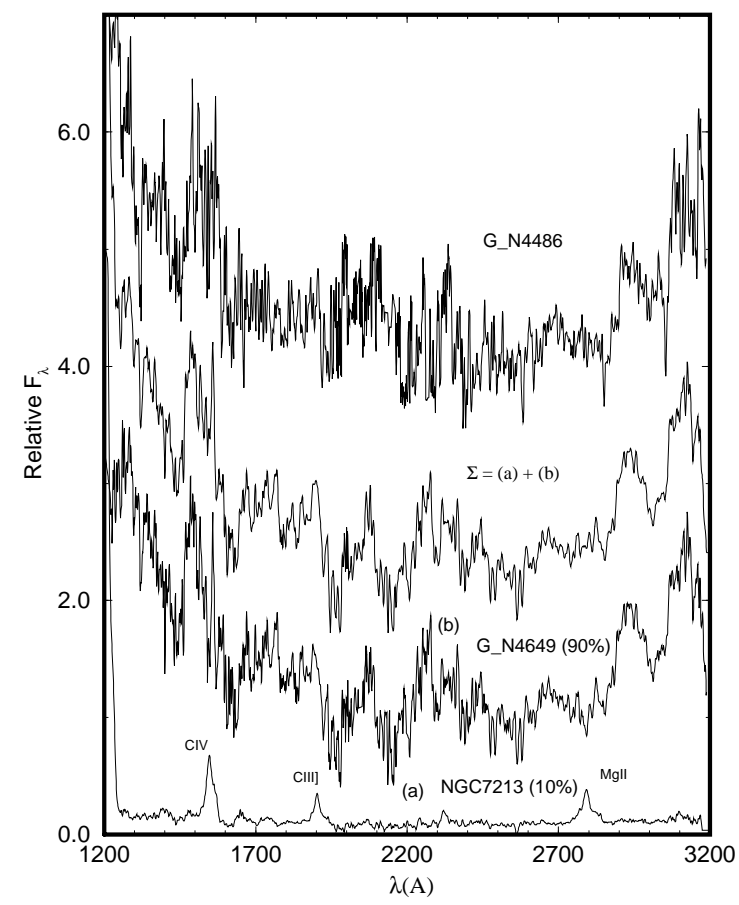

Fig. 16. The spectrum of the strong LINER group G_N4486 can be reproduced by combining those of the Seyfert 1/LINER NGC 7213 and the galaxy group G_N4649; proportions are, respectively, $10 \%$ and $90 \%$ of the flux at $\lambda 2646 \AA$. Flux in $F_{\lambda}$ units, normalised at $\lambda 2646 \AA$. Constants have been added to the spectra for clarity purposes, except for the bottom one

presentation was found by using a combination of typical disk (age $\approx 10-2000 \mathrm{Myr}$ ) and bulge populations, which are reddened by the strong dust lane in this galaxy; the best result was obtained for an internal reddening correction using the SMC extinction law and $E(B-V)_{i}=0.30$. The nuclear regions, in this case, do not contribute significantly to the UV spectrum;

vii) we investigated the possibility of a mini-Seyfert nucleus being present in the groups containing LINERs: for G_N3998 we found that a combination of $20 \%$ flux contribution at $\lambda 2646 \AA$ from the spectrum of the Seyfert 1/LINER galaxy NGC 7213 and $80 \%$ from the UV turnup group G_N4472, reproduces well the overall spectrum of the group; for G_N4486, we found that a good representation requires a $10 \%$ flux contribution from NGC 7213 and 90\% from G_N4649. In both groups the UV turnup is not caused by the mini-Seyfert far-UV continuum.

Acknowledgements. We warmly thank Dr. T. StorchiBergmann for providing us with the computer files for the SMC and LMC extinction curves, and Dr. C. Winge for helpful discussions. We are indebted to the referee, Dr. L. Buson, for interesting remarks. C.B., E.B. and M.G.P. acknowledge CNPq 
and FINEP for partially supporting this work; D.A. acknowledges the support of the Canada-France-Hawaii Telescope as a visiting astronomer.

\section{References}

Antonucci R.R.J., 1993, ARA\&A 31, 473

Arp H.C., Madore B.F., 1987, A Catalague of Southern Peculiar Galaxies and Associations. Cambridge University Press

Bertola F., Capaccioli M., Holm A.V., Oke J.B., 1980, ApJ 237, L65

Bettoni D., Buson L.E., 1987, A\&AS 67, 341

Bica E., Alloin D., 1986, A\&A 166, 83

Bica E., Alloin D., 1987a, A\&A 186, 49

Bica E., Alloin D., 1987b, A\&AS 70, 281

Bica E., 1988, A\&A 195, 76

Bica E., Alloin D., 1988, A\&A 192, 98

Bica E., Pastoriza M.G., Maia M., da Silva L.A., Dottori H., 1991, AJ 102, 1702

Bica E., Bonatto C., Pastoriza M.G., Alloin D., 1995, A\&A (accepted)

Bonatto C., Bica E., Alloin D., 1989, A\&A 226, 23

Bonatto C., Bica E., Alloin D., 1995, A\&AS 112, 71 (Paper I)

Burstein D., Bertola F., Buson L.M., Faber S.M., Lauer T.R., 1988, ApJ 328, 440

Buson L.M., Sadler E.M., Zeilinger W.W., et al., 1993, A\&A 280, 409

Caldwell N., 1984, PASP 96, 287

Crenshaw D.M., Bruegman O.W., Norman D.J., 1990, PASP 102, 463

Dahari O., 1985, ApJS 57, 643

Davies R.L., Burstein D., Dressler A., Faber S.M., Lynden-Bell D., Terlevich R.J., Wegner G., 1987, ApJS 64, 581

de Vaucouleurs G., de Vaucouleurs A., Corwin H.G. Jr., et al., 1991, Third Reference Catalogue of Bright Galaxies. Springer-Verlag, New York (RC3)
Filippenko A.V., Sargent W.L.W., 1985, ApJS 57, 503

Fitzpatrick E.L., 1986, AJ 92, 1068

Gelderman R., Whittle M., 1994, ApJS 91, 491

Goudfrooij P., Hansen L., Jørgensen H.E., Nørgaard-Nielsen H.U., 1994, A\&AS 105, 341

Gregg M.D., 1989, ApJS 69, 217

Heckman T.M., Balick B., Crane P.C., 1980, A\&AS 40, 295

Hewitt A., Burbidge G., 1993, ApJS 87, 451

Kinney A.L., Bohlin R.C., Calzetti D., Panagia N., Wyse R.F.G., 1993, ApJS 86, 5

Kinney A.L., Calzetti D., Bica E., Storchi-Bergmann T., 1994, ApJ 429, 172

Lauberts A., 1982, The ESO/Uppsala Survey of the ESO (B) Atlas. Munich: European Southern Observatory

Longo G., Capaccioli M., Ceriello A., 1991, A\&AS 90, 375

Mazzarella J.M., Balzano V.A., 1986, ApJS 62, 751

Oke J.B., Bertola F., Capaccioli M., 1981, ApJ 243, 453

Osterbrock D.E., Martel A., 1993, ApJ 414, 552

Pastoriza M.G., 1975, Ap\&SS 33, 173

Prévot M.L., Lequeux J., Maurice E., Prévot L., RoccaVolmerange B., 1984, A\&A 132, 389

Rosa M., Joubert M., Benvenutti P., 1984, A\&AS 57, 361

Sandage A., Tammann G.A., 1981, The Revised Shapley-Ames Catalog. Carnegie Inst. Washington, Publ. No. 635

Schmidt A.A., Bica E., Alloin D., 1990, MNRAS 243, 620

Seaton M.J., 1979, MNRAS 187, 73

Sérsic J.L., Carranza G., Pastoriza M.G., 1972, Ap\&SS 19, 469

Stirpe G.M., et al., 1994, A\&A 285, 857

Storchi-Bergmann T., Kinney A.L., Challis, P. 1995, ApJS 98, 103

Terlevich R.J., Melnick J., Masegosa J., Moles M., Copetti M.V., 1991, A\&AS 91, 285

Véron-Cetty M.-P., Véron P., 1991, European Southern Observatory, Scientific Report 5th Edition

Wanders I., et al., 1993, A\&A 269, 39 